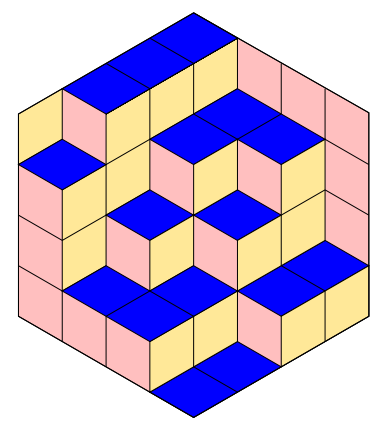

ALGEBRAIC COMBINATORICS

Sho Matsumoto \& Piotr Śniady

Linear versus spin: representation theory of the symmetric groups

Volume 3, issue 1 (2020), p. 249-280.

<http://alco.centre-mersenne.org/item/ALCO_2020__3_1_249_0>

(C) The journal and the authors, 2020.

Some rights reserved.

(c) BY This article is licensed under the

Creative Commons ATtribution 4.0 InTERnational License.

http://creativecommons.org/licenses/by/4.0/

Access to articles published by the journal Algebraic Combinatorics on the website http://alco.centre-mersenne.org/ implies agreement with the Terms of Use (http://alco.centre-mersenne.org/legal/).

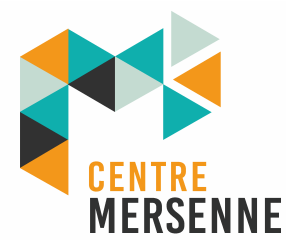

Algebraic Combinatorics is member of the Centre Mersenne for Open Scientific Publishing www.centre-mersenne.org 


\title{
Linear versus spin: representation theory of the symmetric groups
}

\author{
Sho Matsumoto \& Piotr Śniady
}

\begin{abstract}
We relate the linear asymptotic representation theory of the symmetric groups to its spin counterpart. In particular, we give explicit formulas which express the normalized irreducible spin characters evaluated on a strict partition $\xi$ with analogous normalized linear characters evaluated on the double partition $D(\xi)$. We also relate some natural filtration on the usual (linear) Kerov-Olshanski algebra of polynomial functions on the set of Young diagrams with its spin counterpart. Finally, we give a spin counterpart to Stanley formula for the characters of the symmetric groups in terms of counting maps.
\end{abstract}

\section{INTRODUCTION}

Spin representation theory of the symmetric groups is a younger sibling of the usual linear representation theory. In the past, development of the spin sibling took a path parallel to that of the linear sibling (often with a decades long delay): the spin versions of the notions and relationships were modelled after their linear analogues but there were somewhat distinct and they had to be developed from scratch. The paths of the developments of the siblings did not really cross: there were not many results which would directly link these two realms.

In the current paper we consider a setup in which there is a direct link between the spin and the linear representation theory of the symmetric groups. In fact we claim that the spin representation theory (or at least some of its aspects) can be derived from its linear counterpart.

Before we state our results we shall recall some basic concepts of the spin representation theory of the symmetric groups. For more details and bibliographic references refer to $[38,18,15]$.

\subsection{SPIN REPRESENTATION THEORY OF THE SYMMETRIC GROUPS.}

1.1.1. Linear representations. Recall that a linear representation of a finite group $G$ is a group homomorphism $\psi: G \rightarrow \mathrm{GL}(V)$ to the group of linear transformations $\mathrm{GL}(V)$ of some finite-dimensional complex vector space $V$.

Manuscript received 23rd November 2018, revised 8th July 2019, accepted 1st August 2019.

KEYwORDs. Projective representations of the symmetric groups, linear representations of the symmetric groups, asymptotic representation theory, Stanley character formula.

ACKNOWLEDGEMENTS. Research of Sho Matsumoto was supported by JSPS KAKENHI Grant Number 17K05281. Research of Piotr Śniady was supported by Narodowe Centrum Nauki, grant number $2017 / 26 / \mathrm{A} / \mathrm{ST} 1 / 00189$. 
1.1.2. Projective representations. A projective representation of a finite group $G$ is a group homomorphism $\psi: G \rightarrow \operatorname{PGL}(V)$ to the group of projective linear transformations PGL $(V)=\mathrm{GL}(V) / \mathbb{C}^{\times}$of the projective space $P(V)$ for some finite-dimensional complex vector space $V$. Equivalently, a projective representation can be viewed as a map $\phi: G \rightarrow \mathrm{GL}(V)$ to the general linear group with the property that

$$
\phi(x) \phi(y)=c_{x, y} \phi(x y)
$$

holds true for all $x, y \in G$ for some non-zero scalar $c_{x, y} \in \mathbb{C}^{\times}$.

Each irreducible linear representation $\psi: G \rightarrow \mathrm{GL}(V)$ gives rise to its projective version $\psi: G \rightarrow \operatorname{PGL}(V)$. The irreducible projective representations which cannot be obtained in this way are called irreducible spin representations and are in the focus of the current paper.

1.1.3. Spin symmetric group and spin characters. The spin group $\widetilde{\mathfrak{S}}_{n}[29]$ is a double cover of the symmetric group:

$$
1 \longrightarrow \mathbb{Z}_{2}=\{1, z\} \longrightarrow \widetilde{\mathfrak{S}}_{n} \longrightarrow \mathfrak{S}_{n} \longrightarrow 1 \text {. }
$$

More specifically, it is the group generated by $t_{1}, \ldots, t_{n-1}, z$ subject to the relations:

$$
\begin{aligned}
z^{2} & =1, & & \\
z t_{i} & =t_{i} z, & & \\
\left(t_{i} t_{i+1}\right)^{3} & =z & & \text { for } i \in[n-1], \\
t_{i} t_{j} & =z t_{j} t_{i} & & \text { for } i \in[n-2],
\end{aligned}
$$

we use the convention that $[k]=\{1, \ldots, k\}$. Under the mapping $\widetilde{\mathfrak{S}}_{n} \rightarrow \mathfrak{S}_{n}$ the generators $t_{1}, \ldots, t_{n-1}$ are mapped to the Coxeter tranpositions $(1,2),(2,3), \ldots,(n-$ $1, n) \in \mathfrak{S}_{n}$.

The main advantage of the spin group comes from the fact that any projective representation $\psi: \mathfrak{S}_{n} \rightarrow \operatorname{PGL}(V)$ of the symmetric group can be lifted to a linear representation $\widetilde{\psi}: \widetilde{\mathfrak{S}}_{n} \rightarrow \mathrm{GL}(V)$ of the spin group so that the following diagram commutes:

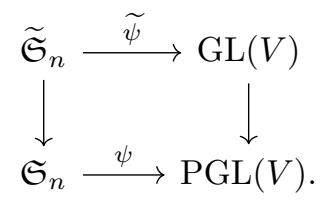

In this way the projective representation theory of $\mathfrak{S}_{n}$ is equivalent to the linear representation theory of $\widetilde{\mathfrak{S}}_{n}$ which allows to speak about the characters.

The irreducible spin representations of $\mathfrak{S}_{n}$ turn out to correspond to irreducible linear representations of the spin group algebra $\mathbb{C S}_{n}^{-}:=\mathbb{C}_{n} /\langle z+1\rangle$ which is the quotient of the group algebra $\mathbb{C} \widetilde{\mathfrak{S}}_{n}$ by the ideal generated by $(z+1)$.

1.1.4. Partitions. An integer partition of $n \geqslant 0$ (often referred to as Young diagram with $n$ boxes) is a finite sequence $\lambda=\left(\lambda_{1}, \ldots, \lambda_{\ell}\right)$ of positive integers (called parts) which is weakly decreasing $\lambda_{1} \geqslant \cdots \geqslant \lambda_{\ell}$ and such that $n=|\lambda|=\lambda_{1}+\cdots+\lambda_{\ell}$. The set of integer partitions of a given integer $n \geqslant 0$ will be denoted by $\mathcal{P}_{n}$, and the set of all integer partitions by $\mathcal{P}=\bigsqcup_{n \geqslant 0} \mathcal{P}_{n}$. We sometimes write $\lambda \vdash n$ instead of $\lambda \in \mathcal{P}_{n}$.

An integer partition is odd if all of its parts are odd integers; we denote by $\mathcal{O P}$ the set of odd partitions and by $\mathcal{O P}$. the set of odd partitions of a given integer $n \geqslant 0$.

An integer partition $\xi=\left(\xi_{1}, \ldots, \xi_{\ell}\right)$ is strict if it has no repeated entries; in other words the corresponding sequence $\xi_{1}>\cdots>\xi_{\ell}$ is strictly decreasing. We denote 
by $\mathcal{S P}$ the set of strict partitions and by $\mathcal{S P} \mathcal{P}_{n}$ the set of strict partitions of a given integer $n \geqslant 0$.

We visualize partitions as Young diagrams drawn in the French convention and strict partitions as shifted Young diagrams, cf. Figure 1.
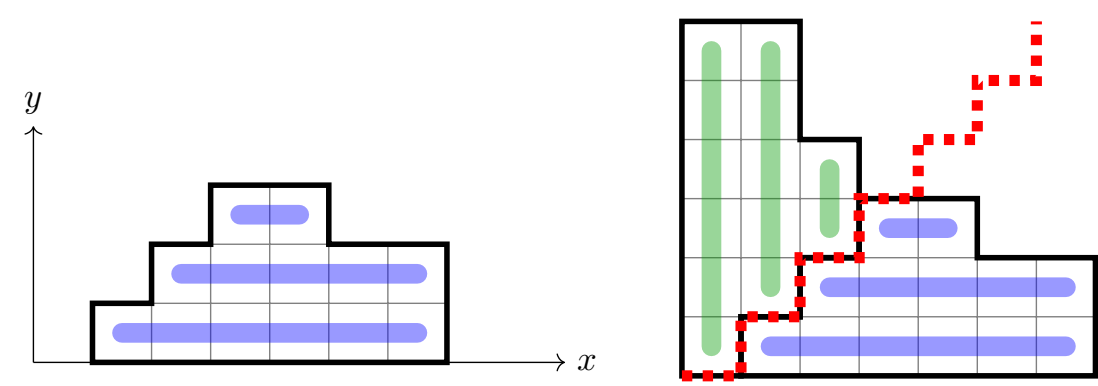

FIGURE 1. Strict partition $\xi=(6,5,2)$ shown as a shifted Young diagram and its double $D(\xi)=(7,7,5,3,2,2)$.

1.1.5. Spin characters. Schur [29] proved that, roughly speaking ${ }^{(1)}$, the conjugacy classes of $\widetilde{\mathfrak{S}}_{n}$ which are non-trivial from the viewpoint of the spin character theory are indexed by the elements of the set $\mathcal{O P}_{n}$, i.e. by the odd partitions of $n$.

Schur also proved that, roughly speaking ${ }^{(1)}$, the irreducible spin representations of $\widetilde{\mathfrak{S}}_{n}$ are indexed by the elements of the set $\mathcal{S P}_{n}$, i.e. by strict partitions of $n$

For an odd partition $\pi \in \mathcal{O P} \mathcal{P}_{n}$ (which corresponds to a conjugacy class of $\widetilde{\mathfrak{S}}_{n}$ ) and a strict partition $\xi \in \mathcal{S P} \mathcal{P}_{n}$ (which corresponds to its irreducible spin representation) we denote by $\phi^{\xi}(\pi)$ the corresponding spin character (for some fine details related to this definition we refer to Section 2).

1.2. Normalized Characters. The usual way of viewing the linear characters $\chi^{\lambda}(\pi)$ of the symmetric group $\mathfrak{S}_{n}$ is to fix the irreducible representation $\lambda$ and to consider the character as a function of the conjugacy class $\pi$. The dual approach, initiated by Kerov and Olshanski [17], suggests to do the opposite: fix the conjugacy class $\pi$ and to view the character as a function of the irreducible representation $\lambda$. In order for this approach to be successful one has to choose the most convenient normalization constants which we review in the following.

Following Kerov and Olshanski [17], for a fixed integer partition $\pi$ the corresponding normalized linear character on the conjugacy class $\pi$ is the function on the set of all Young diagrams given by

$$
\mathrm{Ch}_{\pi}(\lambda):= \begin{cases}n^{\downarrow k} \frac{\chi^{\lambda}\left(\pi \cup 1^{n-k}\right)}{\chi^{\lambda}\left(1^{n}\right)} & \text { if } n \geqslant k, \\ 0 & \text { otherwise }\end{cases}
$$

where $n=|\lambda|$ and $k=|\pi|$ and $n^{\downarrow k}=n(n-1) \cdots(n-k+1)$ denotes the falling power. Above, for partitions $\lambda, \sigma \vdash n$ we denote by $\chi^{\lambda}(\sigma)$ the irreducible linear character of the symmetric group which corresponds to the Young diagram $\lambda$, evaluated on any permutation with the cycle decomposition given by $\sigma$. For more context we refer to the monographs [25, Section 7.3] and [14, Eq. (2.21)].

\footnotetext{
${ }^{(1)}$ For a precise statement see Section 2.
} 
Following Ivanov [15, 16], for a fixed odd partition $\pi \in \mathcal{O P}$ the corresponding normalized spin character is the function on the set of all strict partitions given by

$$
\mathrm{Ch}_{\pi}^{\text {spin }}(\xi):= \begin{cases}n^{\downarrow k} 2^{\frac{k-\ell(\pi)}{2}} \frac{\phi^{\xi}\left(\pi \cup 1^{n-k}\right)}{\phi^{\xi}\left(1^{n}\right)} & \text { if } n \geqslant k, \\ 0 & \text { otherwise, }\end{cases}
$$

where $n=|\xi|, k=|\pi|$, and $\ell(\pi)$ denotes the number of parts of $\pi$.

In the following we shall discuss briefly some heuristics behind these definitions.

Both types of normalized characters (2) and (3) involve evaluation of the irreducible (linear or spin) character on the partition $\pi \cup 1^{n-k}$, i.e. on the partition $\pi$ augmented by the appropriate number of parts, all equal to 1 .

In the case of the linear characters this operation is motivated by considering the infinite sequence of inclusions of the symmetric groups

$$
\mathfrak{S}_{1} \subseteq \mathfrak{S}_{2} \subseteq \mathfrak{S}_{3} \subseteq \cdots
$$

which corresponds to viewing the symmetric group $\mathfrak{S}_{n}$ as the set of these permutations of the set $\mathbb{N}=\{1,2, \ldots\}$ which are equal to the identity outside of $[n]$. Thanks to these inclusions, for $k \leqslant n$ any character of $\mathfrak{S}_{n}$ is also a function on its subgroup $\mathfrak{S}_{k}$. Since the inclusion $\mathfrak{S}_{k} \subseteq \mathfrak{S}_{n}$ is provided by augmenting any given permutation by additional $n-k$ fixpoints, on the level of the cycle lengths this corresponds to replacing any given partition $\pi \vdash k$ by $\pi \cup 1^{n-k} \vdash n$, just as it happens in (2).

In the case of the spin groups the analogous sequence of inclusions

$$
\widetilde{\mathfrak{S}}_{1} \subseteq \widetilde{\mathfrak{S}}_{2} \subseteq \widetilde{\mathfrak{S}}_{3} \subseteq \cdots
$$

is naturally provided by considering first the infinite group generated by the elements $z, t_{1}, t_{2}, \ldots$ which fulfil the relations from Section 1.1.3 and then viewing $\widetilde{\mathfrak{S}}_{n}$ as its subgroup generated by $z, t_{1}, \ldots, t_{n-1}$. It turns out that also in this spin context the inclusion $\widetilde{\mathfrak{S}}_{k} \subseteq \widetilde{\mathfrak{S}}_{n}$ is provided by replacing any given strict partition $\pi \in \mathcal{S P}_{k}$ by $\left(\pi \cup 1^{n-k}\right) \in \mathcal{S} \mathcal{P}_{n}$, see Section 2.2 for more details.

It is more challenging to motivate quickly the specific choice of the normalization constants in (2) and (3). In the case of the linear characters the combinatorial factor $n^{\downarrow k}$ may be interpreted heuristically as the number of ways the smaller symmetric group $\mathfrak{S}_{k}$ can be embedded in the larger symmetric group $\mathfrak{S}_{n}$ or, more precisely, the number of ways any $k$-element set can be embedded in an $n$-element set. In the case of the spin characters a heuristic explanation is even more challenging; probably it can be motivated by the work of Ivanov [15]. The ultimate criterion for verifying that the normalization constants in the definitions of the normalized characters were selected in the right way is postponed to Sections 1.6.1 and 1.6.2. Roughly speaking, thanks to the right choice of the normalization both $\mathrm{Ch}_{\pi}\left(\lambda_{1}, \lambda_{2}, \ldots\right)$ and $\mathrm{Ch}_{\pi}^{\text {spin }}\left(\xi_{1}, \xi_{2}, \ldots\right)$ can be identified with certain polynomials in the variables $\lambda_{1}, \lambda_{2}, \ldots$ (respectively, $\left.\xi_{1}, \xi_{2}, \ldots\right)$.

Our goal in this paper is to find relationships between the family $\left(\mathrm{Ch}_{\pi}\right)$ of linear characters and its spin counterpart $\left(\mathrm{Ch}_{\pi}^{\text {spin }}\right)$. This quite general goal can take various more specific incarnations which we will discuss in the following.

1.3. The main Result 1. Characters: linear vs spin. Firstly, we might look for some explicit algebraic formulas which express the linear characters $\left(\mathrm{Ch}_{\pi}\right)$ in terms of the spin characters $\left(\mathrm{Ch}_{\pi}^{\text {spin }}\right)$ and vice versa.

The first step in this direction is to relate the set $\mathcal{S P}$ of strict partitions to the set $\mathcal{P}$ of partitions which are, respectively, the domain of the linear characters $\left(\mathrm{Ch}_{\pi}\right)$ and the domain of the spin characters $\left(\mathrm{Ch}_{\pi}^{\text {spin }}\right)$. More specifically, for a strict partition $\xi \in \mathcal{S P}_{n}$ we consider its double $D(\xi) \in \mathcal{P}_{2 n}$. Graphically, $D(\xi)$ corresponds to a Young 
diagram obtained by arranging the shifted Young diagram $\xi$ and its 'transpose' so that they nicely fit along the 'diagonal', cf. Figure 1, see also [21, Example I.1.9(a)].

In Section 3 we will show several formulas which relate linear and spin characters. The following is a particularly simple special case which nevertheless gives a flavour of the general case.

THeOREM 1.1 (The main result for characters, special case).

For any odd integer $k \geqslant 1$ and any strict partition $\xi \in \mathcal{S P}$

$$
\mathrm{Ch}_{k}(D(\xi))=2 \mathrm{Ch}_{k}^{\text {spin }}(\xi) .
$$

We will prove this result (in wider generality) in Section 3.2. The results of this type reduce the problem of calculating the spin characters to its linear counterpart for which often more tools are available $[28,11,13,3,27,7]$.

\subsection{The main Result 2. Spin version of Stanley character formula.}

1.4.1. Colorings. Let $\sigma_{1}, \sigma_{2} \in \mathfrak{S}_{k}$ be permutations and let $\lambda \in \mathcal{P}$ be a Young diagram. Following [11], we say that $\left(f_{1}, f_{2}\right)$ is a coloring of $\left(\sigma_{1}, \sigma_{2}\right)$ which is compatible with $\lambda$ if the following two conditions are fulfilled:

- $f_{i}: C\left(\sigma_{i}\right) \rightarrow \mathbb{Z}_{+}$is a function on the set of cycles of $\sigma_{i}$ for each $i \in\{1,2\}$; we view the values of $f_{1}$ as columns of $\lambda$ and the values of $f_{2}$ as rows;

- whenever $c_{1} \in C\left(\sigma_{1}\right)$ and $c_{2} \in C\left(\sigma_{2}\right)$ are cycles which are not disjoint $\left(c_{1} \cap\right.$ $\left.c_{2} \neq \varnothing\right)$, the box with Cartesian coordinates $\left(f_{1}\left(c_{1}\right), f_{2}\left(c_{2}\right)\right)$ belongs to $\lambda$.

We denote by $N_{\sigma_{1}, \sigma_{2}}(\lambda)$ the number of such colorings of $\left(\sigma_{1}, \sigma_{2}\right)$ which are compatible with $\lambda$.

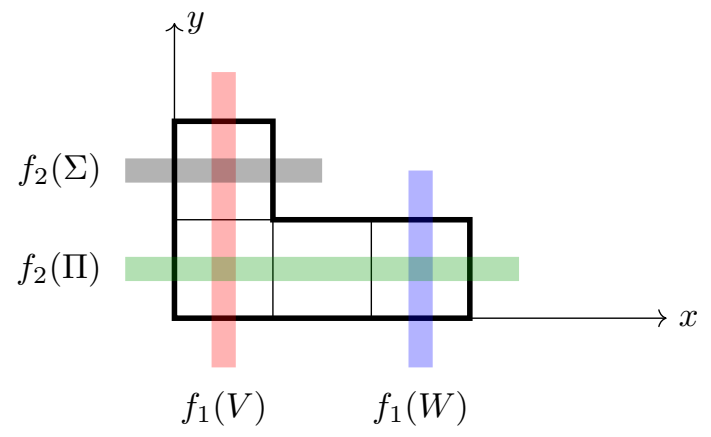

Figure 2. Graphical representation of the coloring (5) of the permutations (4) which is compatible with the Young diagram $\lambda=(3,1)$.

EXAMPLE 1.2. Let

$$
\sigma_{1}=\underbrace{(1,5,4,2)}_{V} \underbrace{(3)}_{W}, \quad \sigma_{2}=\underbrace{(2,3,5)}_{\Pi} \underbrace{(1,4)}_{\Sigma} .
$$

There are three pairs of cycles $\left(\sigma_{1}, \sigma_{2}\right) \in C\left(\sigma_{1}\right) \times C\left(\sigma_{2}\right)$ with the property that $\sigma_{1}$ and $\sigma_{2}$ are not disjoint, namely $(V, \Pi),(V, \Sigma),(W, \Pi)$. It is now easy to check graphically (cf. Figure 2$)$ that $\left(f_{1}, f_{2}\right)$ given by

$$
f_{1}(V)=1, \quad f_{1}(W)=3, \quad f_{2}(\Pi)=1, \quad f_{2}(\Sigma)=2
$$

is indeed an example of a coloring compatible with $\lambda=(3,1)$.

By considering four possible choices for the values of $f_{2}$ and counting the choices for the values of $f_{1}$ one can verify that

$$
N_{\sigma_{1}, \sigma_{2}}(\lambda)=3^{2}+3+1+1=14 \quad \text { for } \lambda=(3,1) .
$$


1.4.2. Linear Stanley character formula. We will identify a given integer partition $\pi=\left(\pi_{1}, \ldots, \pi_{\ell}\right) \vdash k$ with an arbitrary permutation $\pi \in \mathfrak{S}_{k}$ with the corresponding cycle structure. For example, we may take

$$
\pi=\left(1,2, \ldots, \pi_{1}\right)\left(\pi_{1}+1, \pi_{1}+2, \ldots, \pi_{1}+\pi_{2}\right) \cdots \in \mathfrak{S}_{k} .
$$

The following result turned out in the past to be a convenient tool for studying asymptotic [11] and enumerative problems [7] of the (linear) representation theory of the symmetric groups.

Theorem 1.3 ([11]). For any partition $\pi \in \mathcal{P}_{k}$ and any Young diagram $\lambda \in \mathcal{P}$

$$
\mathrm{Ch}_{\pi}(\lambda)=\sum_{\substack{\sigma_{1}, \sigma_{2} \in \mathfrak{S}_{k} \\ \sigma_{1} \sigma_{2}=\pi}}(-1)^{\sigma_{1}} N_{\sigma_{1}, \sigma_{2}}(\lambda) .
$$

1.4.3. Spin Stanley character formula. For $\sigma_{1}, \sigma_{2} \in \mathfrak{S}_{k}$ we denote by $\left|\sigma_{1} \vee \sigma_{2}\right|$ the number of orbits in the set $[k]=\{1, \ldots, k\}$ under the action of the group $\left\langle\sigma_{1}, \sigma_{2}\right\rangle$ generated by $\sigma_{1}, \sigma_{2}$. Equivalently, it is the number of blocks of the set-partition $\sigma_{1} \vee \sigma_{2}$ (which is the minimal set-partition with respect to the refinement order which is greater than each of the two set-partitions which correspond to the cycles of $\sigma_{1}$ and $\left.\sigma_{2}\right)$.

One of the main results of the current paper is the following spin analogue of Theorem 1.3 .

Theorem 1.4 (The main result: spin Stanley character formula). For any odd partition $\pi \in \mathcal{O P}_{k}$ and any strict partition $\xi \in \mathcal{S P}$

$$
\mathrm{Ch}_{\pi}^{\text {spin }}(\xi)=\sum_{\substack{\sigma_{1}, \sigma_{2} \in \mathfrak{G}_{k} \\ \sigma_{1} \sigma_{2}=\pi}} \frac{1}{2^{\left|\sigma_{1} \vee \sigma_{2}\right|}}(-1)^{\sigma_{1}} N_{\sigma_{1}, \sigma_{2}}(D(\xi)) .
$$

The proof is postponed to Section 4.

1.4.4. Application: combinatorics of spin Kerov polynomials and Stanley polynomials. In the context of the usual (linear) asymptotic representation theory of the symmetric groups it is convenient to parametrize the set of Young diagrams by free cumulants [1]. Each linear character $\mathrm{Ch}_{\pi}$ can then be expressed uniquely as a polynomial (called Kerov character polynomial) in these free cumulants. Kerov polynomials turned out to have a very rich structure from the viewpoint of enumerative and algebraic combinatorics $[2,13,3,27,7,31]$. Combinatorics of Kerov polynomials is intimately related to multirectangular coordinates of Young diagrams and the corresponding Stanley character polynomials, see Section 6.

Recently De Stavola [5] and the first-named author [22] initiated investigation of spin counterparts of these notions. In a future work we will present applications of Theorem 1.4 to combinatorics of spin Kerov polynomials and spin Stanley character polynomials.

1.4.5. Application: bounds on spin characters. The following character bound is a spin version of an analogous result for the linear characters of the symmetric group [11]. It is a direct application of Theorem 1.4 and its proof follows the same line as its linear counterpart [11].

COROLLARY 1.5. There exists a universal constant $a>0$ with the property that for any integer $n \geqslant 1$, any strict partition $\xi=\left(\xi_{1}, \xi_{2} \ldots\right) \in \mathcal{S P}_{n}$, and any odd partition $\pi \in \mathcal{O P}_{n}$

$$
\left|\frac{\phi^{\xi}(\pi)}{\phi^{\xi}\left(1^{n}\right)}\right|<\left[a \max \left(\frac{\xi_{1}}{n}, \frac{n-\ell(\pi)}{n}\right)\right]^{n-\ell(\pi)} .
$$


Several asymptotic results about (random) Young diagrams and tableaux which use the inequality from [11] can be generalized in a rather straightforward way to (random) shifted Young diagrams and shifted tableaux thanks to Corollary 1.5. A good example is provided by the results of Dousse and Féray about the asymptotics of the number of skew standard Young tableaux of prescribed shape [9] which can be generalized in this way to asymptotics of the number of shifted skew standard Young tableaux.

1.4.6. Open problem: towards irreducible representations of spin groups. The proof of the linear Stanley formula (6) presented in [11] was found in the following way. We attempted to reverse-engineer the right-hand side of (6) and to find

- some natural vector space $V$ with the basis indexed by combinatorial objects; the space $V$ should be a representation of the symmetric group $\mathfrak{S}_{n}$ with $n:=|\lambda|$, and

- a projection $\Pi: V \rightarrow V$ such that $\Pi$ commutes with the action of $\mathfrak{S}_{n}$ and such that it image $\Pi V$ is an irreducible representation of $\mathfrak{S}_{n}$ which corresponds to the specified Young diagram $\lambda$,

in such a way that the corresponding character of $\Pi V$ would coincide with the righthand side of $(6)$.

Our attempt was successful: one could consider a vector space $V$ with the basis indexed by fillings of the boxes of $\lambda$ with the numbers from $[n]$ with the action of $\mathfrak{S}_{n}$ given by pointwise relabelling of the values in the boxes. The projection $\Pi$ turned out to be the Young symmetrizer with the action given by shuffling of the boxes in the rows and columns of $\lambda$. The resulting representation $\Pi V$ clearly coincides with the Specht module, which concluded the proof in [11].

The structure of the right-hand side of (7) (as well as the right-hand side of (8)) might be an indication that an analogous reverse-engineering process could be applied to the spin case. The result would be a very explicit construction of the irreducible spin representations which would be an alternative to the somewhat complicated approach of Nazarov [26].

1.5. Stanley formulas And EnUmeration of MAPs. Some readers may have aesthetic objections against Equation (7) related to the somewhat ugly factor $\frac{1}{2^{\left|\sigma_{1} \vee \sigma_{2}\right|}}$ on the right-hand side. Our remedy to this issue is Theorem 1.7 which avoids such factors. This result is just a reformulation of Theorem 1.4 in the language of nonoriented maps which are orientable. We start by introducing the notations which are necessary to state this result.

1.5.1. Maps. We recall that a map [19] is defined as a graph $\mathcal{G}$ drawn on a surface $\Sigma$ without boundary in such a way that each connected component of $\Sigma \backslash \mathcal{G}$ (called face) is homeomorphic to an open disc. In the literature one often adds the requirement that the surface $\Sigma$ is connected; we will not impose such a restriction.

Each map which we will consider is a bicolored map which means that the set of its vertices $\mathcal{V}=\mathcal{V}_{\circ} \sqcup \mathcal{V}_{\bullet}$ is canonically decomposed into two classes (usually called white and black vertices), with edges connecting only vertices of the opposite colors. Let $F_{1}, \ldots, F_{\ell}$ be the faces of the map; for the above reason of bicoloration, each face $F_{i}$ is a polygon which consists of an even number (say, $2 \pi_{i}$ ) of edges, with neighbouring vertices painted in the alternating colours. The integer partition $\pi=\left(\pi_{1}, \ldots, \pi_{\ell}\right)$ is called the face-type of the map, see Figure 4.

With this in mind, one can alternatively view a bicolored map as a collection $\mathcal{P}$ of polygons with labelled edges and with the vertices painted in the alternating colours (see Figure 3), together with the information which pairs of the edges should be glued 
together. Note that at first moment it might appear that there are two ways to glue any given pair of edges (these two ways differ by a twist a la Möbius), nevertheless the bicoloration of the vertices makes the choice unique.

1.5.2. Non-oriented maps. The above discussion motivates the following definition. Summation over non-oriented maps with a specified face-type $\pi$ [8, Section 3.4] should be understood as follows: we start by fixing an appropriate collection $\mathcal{P}$ of polygons with labelled edges, bicolored vertices, and with face-type $\pi$. Then we consider all perfect matchings on the set of all edges, and for each such a perfect matching we glue the corresponding pairs of the edges. We sum over the resulting map.
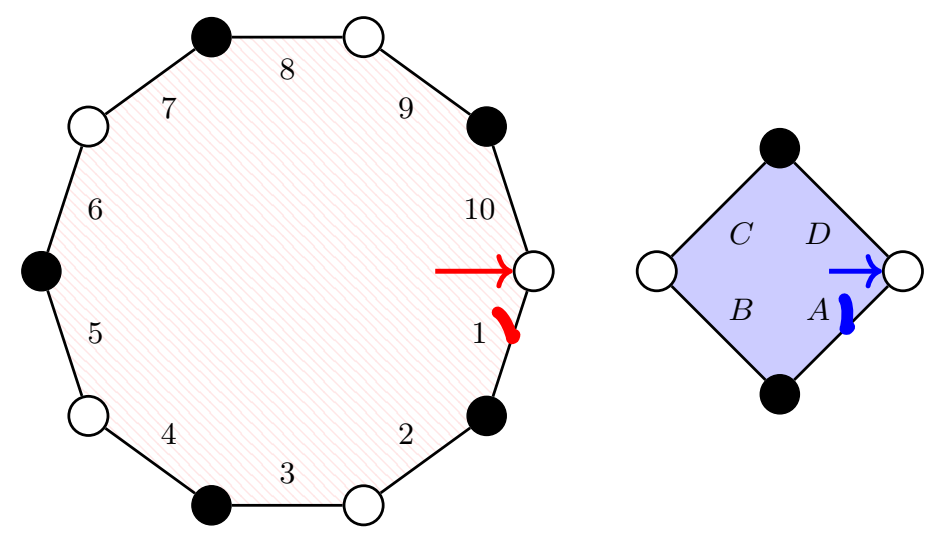

Figure 3. Example of a collection of polygons with face-type $(5,2)$. Arrows (pointing at white vertices between the edges 1 and 10, as well as between $A$ and $D$ ) indicate the roots; harpoons (at the edges 1 and $A$ ) indicate the directions of rotation in each polygon, see Section 5.1 .

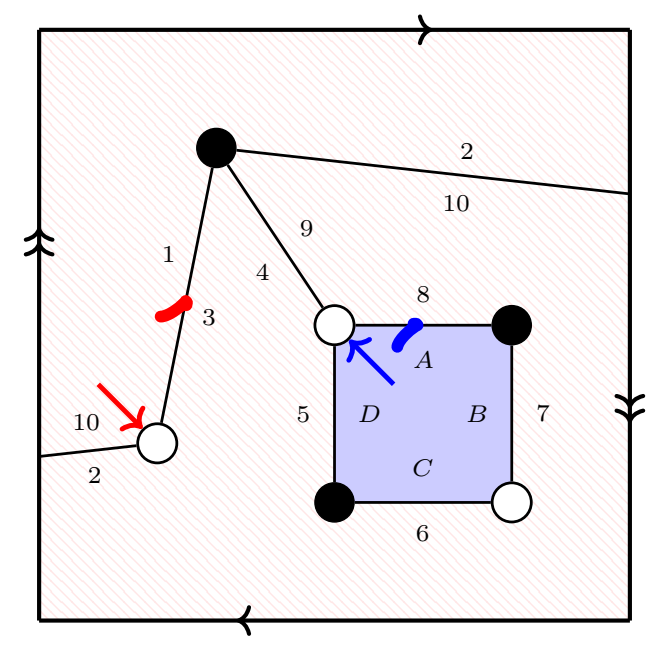

FiguRE 4. Example of a non-oriented map with face-type $(5,2)$ drawn on the projective plane. The left side of the square should be glued with a twist to the right side, as well as bottom to top (also with a twist), as indicated by arrows. This map has been obtained by gluing the edges of the polygons from Figure 3 . 
1.5.3. Colorings revisited. The notion of number of colorings $N_{\sigma_{1}, \sigma_{2}}(\lambda)$ was introduced in Section 1.4.1 in the context of a pair of permutations. We review how to generalize it to maps and, more generally, bicolored graphs.

Let $\mathcal{G}$ be a bicolored graph with the vertex set $\mathcal{V}=\mathcal{V}_{\circ} \sqcup \mathcal{V}_{\bullet}$ and let $\lambda \in \mathcal{P}$ be a Young diagram. We say that $\left(f_{1}, f_{2}\right)$ is a coloring of $\mathcal{G}$ which is compatible with $\lambda$ if the following two conditions are fulfilled:

- $f_{1}: \mathcal{V}_{\circ} \rightarrow \mathbb{Z}_{+}$is a function on the set of white vertices while $f_{2}: \mathcal{V}_{\bullet} \rightarrow \mathbb{Z}_{+}$is a function on the set of black vertices;

- whenever vertices $v_{1} \in \mathcal{V}_{\circ}$ and $v_{2} \in \mathcal{V}_{\bullet}$ are connected by an edge, the box with Cartesian coordinates $\left(f_{1}\left(v_{1}\right), f_{2}\left(v_{2}\right)\right)$ belongs to $\lambda$.

We denote by $N_{\mathcal{G}}(\lambda)$ the number of such colorings of $\mathcal{G}$ which are compatible with $\lambda$.

REMARK 1.6. In Section 5.3 we shall explain how to associate a map $\mathcal{G}$ to a pair $\left(\sigma_{1}, \sigma_{2}\right)$ of permutations; the above definition of $N_{\mathcal{G}}(\lambda)$ is compatible with this correspondence in the sense that

$$
N_{\sigma_{1}, \sigma_{2}}(\lambda)=N_{\mathcal{G}}(\lambda),
$$

where the left-hand side should be understood in the sense of Section 5.3.

1.5.4. Spin Stanley formula and non-oriented, orientable maps. The following is a reformulation of Theorem 1.4.

THEOREM 1.7. For any odd partition $\pi \in \mathcal{O P}_{k}$ and any strict partition $\xi \in \mathcal{S P}$

$$
2^{\ell(\pi)} \mathrm{Ch}_{\pi}^{\text {spin }}(\xi)=\sum_{M}(-1)^{|\pi|-\left|\mathcal{V}_{\circ}(M)\right|} N_{M}(D(\xi))
$$

where the sum runs over non-oriented, orientable bicolored maps with the face-type $\pi$, as in Section 1.5.2.

The proof is postponed to Section 5 .

1.5.5. Stanley formulas and maps. Table 1 summarizes some known and some hypothetical Stanley formulas. It seems that there is a correspondence between some natural functions on the set of (shifted) Young diagrams and natural classes of bicolored maps. Is there some general pattern? Are there some natural classes of maps which are missing in this table?

\subsection{The main Result 3. Kerov-Olshanski algebra: linear vs SPIN.}

1.6.1. Kerov-Olshanski algebra. The usual (linear) Kerov-Olshanski algebra $\mathbb{A}[17$, 14] (also known under the less compact name algebra of polynomial functions on the set of Young diagrams; in the monograph [25, Section 7] it is referred to as IvavovKerov algebra) is an important tool in the (linear) asymptotic representation theory of the symmetric groups. One of its advantages comes from the fact that it can be characterized in several equivalent ways (for example as the algebra $\Lambda^{*}$ of shifted symmetric functions); it also has several convenient linear and algebraic bases which are related to various viewpoints and aspects of the asymptotic representation theory.

For the purposes of the current paper, Kerov-Olshanski algebra

$$
\mathbb{A}:=\operatorname{span}\left\{\mathrm{Ch}_{\pi}: \pi \in \mathcal{P}\right\}
$$

may be defined as the linear span of the normalized linear characters of the symmetric groups. 
Sho Matsumoto \& Piotr Śniady

\begin{tabular}{|c|c|c|}
\hline TYPE OF CHARACTERS & TYPE OF MAPS & REFERENCES \\
\hline $\begin{array}{l}\text { Linear characters } \\
\text { of the symmetric groups }\end{array}$ & Oriented maps & $\begin{array}{l}{[31, \text { Sections } 6.4-6.5],} \\
{[32, \text { Section } 8]}\end{array}$ \\
\hline $\begin{array}{l}\text { Spin characters } \\
\text { of the symmetric groups }\end{array}$ & $\begin{array}{l}\text { Non-oriented maps } \\
\text { which are orientable }\end{array}$ & $\begin{array}{l}\text { Current paper, } \\
\text { Theorem } 1.7\end{array}$ \\
\hline Zonal characters & \multirow{2}{*}{ Non-oriented maps } & \multirow{2}{*}{12, Section 5] } \\
\hline $\begin{array}{l}\text { Symplectic zonal } \\
\text { characters }\end{array}$ & & \\
\hline \multirow[t]{2}{*}{$\begin{array}{l}\text { Top-degree } \\
\text { of Jack characters }\end{array}$} & $\begin{array}{l}\text { Oriented, } \\
\text { connected maps } \\
\text { with arbitrary } \\
\text { face structure }\end{array}$ & {$[33$, Sections 1.4.2-1.4.3] } \\
\hline & $\begin{array}{l}\text { Non-oriented maps } \\
\text { with prescribed } \\
\text { face structure }\end{array}$ & {$[4$, Section 4] } \\
\hline $\begin{array}{l}\text { Jack characters in the } \\
\text { generic case }\end{array}$ & $\begin{array}{l}\text { conjecturally: } \\
\text { non-oriented maps } \\
\text { (counted with some } \\
\text { unknown weight) }\end{array}$ & partial results: [8] \\
\hline
\end{tabular}

TABLE 1. Classes of functions on the set of (shifted) Young diagrams for which some version of Stanley character formula is known (or conjectured in the case of Jack characters) and the corresponding class of maps over which the summation is performed.

1.6.2. Spin Kerov-Olshanski algebra. We define the spin Kerov-Olshanski algebra (maybe Ivanov algebra would be an even better name)

$$
\Gamma:=\operatorname{span}\left\{\mathrm{Ch}_{\pi}^{\text {spin }}: \pi \in \mathcal{S P}\right\}
$$

as the linear span of spin characters [15, Section 6]. Ivanov proved that the elements of $\Gamma$ can be identified with supersymmetric polynomials, thus $\Gamma$ is a unital, commutative algebra.

1.6.3. Double of a function. Kerov-Olshanski algebra: linear vs spin. If $F: \mathcal{P} \rightarrow \mathbb{Q}$ is a function on the set of partitions, we define its double as the function $D^{*} F: \mathcal{S P} \rightarrow \mathbb{Q}$ on the set of strict partitions

$$
\left(D^{*} F\right)(\xi):=F(D(\xi)) \quad \text { for } \xi \in \mathcal{S P}
$$

given by doubling of the argument.

The following simple result reduces some questions about the spin Kerov-Olshanski algebra $\Gamma$ to its linear counterpart $\mathbb{A}$.

THEOREM 1.8. The map

$$
D^{*}: \mathbb{A} / \operatorname{ker} D^{*} \rightarrow \Gamma
$$

is an isomorphism of algebras.

The proof is postponed to Section 8 . 
1.6.4. Filtrations: linear vs spin. In applications it is often convenient to equip KerovOlshanski algebra with this or another filtration which is tailored for the specific asymptotic regime one is interested in; several such filtrations were considered in the literature. In order to stay focused we will concentrate on a specific choice of such a filtration $\mathcal{F}_{0} \subseteq \mathcal{F}_{1} \subseteq \cdots \subseteq \mathbb{A}$ given by

$$
\mathcal{F}_{k}:=\operatorname{span}\left\{\mathrm{Ch}_{\pi}: \pi \in \mathcal{P}, \quad|\pi|+\ell(\pi) \leqslant k\right\},
$$

where $\ell(\pi)=\ell$ denotes the number of parts of $\pi=\left(\pi_{1}, \ldots, \pi_{\ell}\right)$. This specific choice is motivated by investigation of asymptotics of (random) Young diagrams and tableaux in the scaling in which they grow to infinity in such a way that they remain balanced $[1,30]$.

We define its spin counterpart as the family of vector spaces $\mathcal{G}_{0} \subseteq \mathcal{G}_{1} \subseteq \cdots \subseteq \Gamma$ defined by

$$
\mathcal{G}_{k}:=\operatorname{span}\left\{\mathrm{Ch}_{\pi}^{\text {spin }}: \pi \in \mathcal{O} \mathcal{P}, \quad|\pi|+\ell(\pi) \leqslant k\right\} .
$$

Note that $|\pi|+\ell(\pi)$ is always an even integer for any $\pi \in \mathcal{O P}$; it follows therefore that $\mathcal{G}_{2 k+1}=\mathcal{G}_{2 k}$ holds for any integer $k \geqslant 0$. Informally speaking, this means that only the 'even' part of the family $\mathcal{G}_{0} \subseteq \mathcal{G}_{1} \subseteq \cdots \subseteq \Gamma$ contains interesting information.

The following result provides a direct link between the families $\left(\mathcal{F}_{i}\right)$ and $\left(\mathcal{G}_{i}\right)$ via the isomorphism (10). Also its proof (postponed to Section 8) makes use of this isomorphism.

THEOREM 1.9. The family $\left(\mathcal{G}_{k}\right)$ is a filtration on the algebra $\Gamma$.

Furthermore, $\mathcal{G}_{k}=D^{*}\left(\mathcal{F}_{k}\right)$ for any integer $k \geqslant 0$.

1.6.5. Application. Gaussian fluctuations of partitions: linear vs spin. Our motivation for studying the filtration $\left(\mathcal{G}_{i}\right)$ comes from investigation of random strict partitions related to projective asymptotic representation theory of the symmetric groups. Theorem 1.9 plays a prominent role in our forthcoming paper [24] which is devoted to this topic.

A convenient tool for proving Gaussianity of fluctuations of random partitions is approximate factorization property for characters $[30,6]$ which is formulated in the language of certain cumulants. In the case of the linear characters of the symmetric groups this property was known to be true [30].

In the aforementioned forthcoming paper [24] we will show how to reformulate the results of the current paper in a more abstract way which allows to relate the cumulants for linear characters to their spin counterparts. In this way we will show how the approximate factorization property of linear characters of the symmetric groups implies directly its spin counterpart.

1.7. Structure of THE PAPER. Section 2 summarizes some fine issues related to the definition of the spin characters.

In Section 3 we prove our starting tool, Theorem 3.1, which expresses the linear characters $\left(\mathrm{Ch}_{\pi}\right)$ in terms of the spin characters $\left(\mathrm{Ch}_{\pi}^{\text {spin }}\right)$. As a consequence, we also prove Theorem 3.3 which gives roughly the opposite: the spin characters $\left(\mathrm{Ch}_{\pi}^{\text {spin }}\right)$ in terms of the linear characters $\left(\mathrm{Ch}_{\pi}\right)$.

Section 4 is devoted to the proof of Theorem 1.4, i.e. spin Stanley character formula. Section 5 is devoted to the proof of Theorem 1.7, i.e. spin Stanley formula in terms of non-oriented, orientable maps.

In Section 6 we recall the shifted version of multirectangular coordinates and in Section 7 we apply these coordinates for asymptotics of spin characters. In Section 8 we apply these results in order to prove Theorems 1.8 and 1.9 which provide the link between (the filtration on) the linear Kerov-Olshanski algebra and its spin counterpart. 
This article is the full version of a 12-page extended abstract [23] which will be published in the proceedings of the conference Formal Power Series and Algebraic Combinatorics 2019.

\section{SPIN CHARACTERS, SPIN REPRESENTATIONS}

In order to keep the Introduction lightweight we decided to postpone the discussion of some subtle technical issues related to the definition of spin characters and spin representations until the current section. Our presentation is based on [37, 38, 18, 15].

2.1. Conjugacy Classes of $\widetilde{\mathfrak{S}}_{n}$. We denote by $\mathcal{S P}_{n}^{+}$(respectively, $\mathcal{S P}{ }_{n}^{-}$) the set of strict partitions $\xi \in \mathcal{S} \mathcal{P}_{n}$ with the property that $n-\ell(\xi)$ is even (respectively, odd).

For a partition $\pi \vdash n$ we denote by $C_{\pi} \subset \widetilde{\mathfrak{S}}_{n}$ the set of elements of the spin group which are mapped - under the canonical homomorphism $\widetilde{\mathfrak{S}}_{n} \rightarrow \mathfrak{S}_{n}$ - to permutations with the cycle-type given by $\pi$.

Schur [29] proved the following dichotomy for $\pi \vdash n$ :

- if one of the following two conditions is fulfilled:

$-\pi \in \mathcal{O P}_{n}$, or

$-\pi \in \mathcal{S P}_{n}^{-}$

then $C_{\pi}$ splits into a pair of conjugacy classes of $\widetilde{\mathfrak{S}}_{n}$ which will be denoted by $C_{\pi}^{ \pm}$;

- otherwise, $C_{\pi}$ is a conjugacy class of $\widetilde{\mathfrak{S}}_{n}$.

2.2. Conjugacy Classes And spin Characters. Any spin character vanishes on the conjugacy class $C_{\pi}$ which does not split, cf. [37, p. 95]. For this reason, from the viewpoint of the spin character theory only the conjugacy classes $C_{\pi}^{ \pm}$are interesting.

Spin representations are exactly the ones which map the central element $z \in \widetilde{\mathfrak{S}}_{n}$ to $-\mathrm{Id} \in \mathrm{GL}(V)$. Since $C_{\pi}^{-}=z C_{\pi}^{+}$, it follows that the value of any spin character on $C_{\pi}^{-}$is the opposite of its value on $C_{\pi}^{+}$. For this reason, from the viewpoint of the spin character theory the conjugacy classes $C_{\pi}^{-}$are redundant and it is enough to consider the character values only on the conjugacy classes $C_{\pi}^{+}$.

From the viewpoint of the asymptotic representation theory it is natural to consider some sequence of groups together with some natural inclusions; in our case this is the sequence

$$
\widetilde{\mathfrak{S}}_{1} \subset \widetilde{\mathfrak{S}}_{2} \subset \widetilde{\mathfrak{S}}_{3} \subset \cdots
$$

of spin groups. Such a setup allows to relate a conjugacy class of a smaller group to some conjugacy class in the bigger group and, in this way, to evaluate the irreducible characters of the bigger group on the conjugacy classes of the smaller one.

Regretfully, the conjugacy classes $C_{\pi}^{+}$which correspond to $\pi \in \mathcal{S P}_{n}^{-}$do not behave nicely under such inclusions. Indeed, on the level of the symmetric groups the inclusion $\mathfrak{S}_{n} \subset \mathfrak{S}_{n+k}$ corresponds to adding $k$ fixpoints to a given permutation; in other words the set $C_{\pi} \subset \widetilde{\mathfrak{S}}_{n}$ corresponds to $C_{\pi, 1^{k}} \subset \widetilde{\mathfrak{S}}_{n+k}$ and the latter does not split because $\left(\pi, 1^{k}\right) \notin \mathcal{S} \mathcal{P}_{n}$ (at least for $\left.k \geqslant 2\right)$ and $\left(\pi, 1^{k}\right) \notin \mathcal{O} \mathcal{P}_{n}$ (because $n-\ell(\pi)$ is odd which implies that at least one part of $\pi$ is even).

For this reason, for the purposes of the asymptotic representation theory it is enough to consider only the conjugacy classes $C_{\pi}^{+}$for $\pi \in \mathcal{O P}_{n}$.

2.3. IRREDUCIBLE SPIN REPRESENTATIONS. The relationship between strict partitions and the irreducible spin representations of the symmetric groups is not a bijective one. Nevertheless, as we shall discuss below, this non-bijectivity can be ignored to large extent. 
More specifically (see [29, p. 235] and [37, Theorem 7.1]), each $\xi \in \mathcal{S P}_{n}^{+}$corresponds to a single irreducible representation. We denote by its character by $\phi^{\xi}$.

On the other hand, each $\xi \in \mathcal{S P}_{n}^{-}$corresponds to a pair of irreducible spin representations; we denote their characters by $\phi_{+}^{\xi}$ and $\phi_{-}^{\xi}$. These two characters coincide on the conjugacy classes $C_{\pi}^{ \pm}$over $\pi \in \mathcal{O P}_{n}$. For the purposes of the current paper we do not need to evaluate the characters on $C_{\pi}^{ \pm}$for $\pi \in \mathcal{S P}_{n}^{-}$; for this reason we do not have to distinguish between them and we may denote them by the same symbol $\phi^{\xi}$.

2.4. Spin Characters: CONClusion. For all partitions $\xi \in \mathcal{S P}_{n}, \pi \in \mathcal{O P}_{n}$ the value of the projective character

$$
\phi^{\xi}(\pi)=\operatorname{Tr} \psi^{\xi}\left(c^{\pi}\right)
$$

is well defined, where $c^{\pi} \in C_{\pi}^{+}$is a representative of the of the conjugacy class $C_{\pi}^{+}$, cf. [37, Eq. (2.1)].

2.5. Spin Characters and symmetric functions. In the context of the theory of symmetric functions it is more convenient to pass from spin characters $\phi^{\xi}$ to some other quantities which are denoted by $X^{\lambda}$. We shall review them in the following.

Following [21, Section III.7] we denote by $X(t)$ the transition matrix between the basis of Newton's power-sum symmetric functions $\left(p_{\pi}\right)$ and the basis of HallLittlewood functions:

$$
p_{\pi}=\sum_{\lambda} X^{\lambda}(\pi ; t) P_{\lambda}(t) .
$$

For some special choices of the parameter $t$ the quantities $X(t)$ turn out to have interesting interpretations. For example, in the case $t=0$ we recover the linear characters of the symmetric groups $\chi^{\lambda}(\pi)=X^{\lambda}(\pi ; 0)$ with $\lambda, \pi \in \mathcal{P}_{n}$, cf. [21, III.(7.2)].

From the following on we shall concentrate on another special case $t=-1$ for which Hall-Littlewood functions turn out to be related to Schur's $P$ - and $Q$-functions [21, Section III.8]. In this specific setup the defining property (13) of the transition matrix $X=X(-1)$ takes the form

$$
p_{\pi}=\sum_{\xi \in \mathcal{S} \mathcal{P}_{n}} X^{\xi}(\pi) P_{\xi}
$$

for $n=|\pi|$, or equivalently, for $n=|\xi|$

$$
Q_{\xi}=\sum_{\nu \in \mathcal{O P}_{n}} 2^{\ell(\nu)} z_{\nu}^{-1} X^{\xi}(\nu) p_{\nu}
$$

where $P_{\xi}, Q_{\xi}$ are the Schur's $P$ - and $Q$-functions, as in [21, Example III.8.11(c)] and

$$
z_{\pi}=\prod_{j \geqslant 1} j^{m_{j}(\pi)} m_{j}(\pi) !
$$

where $m_{j}(\pi)$ denotes the multiplicity of $j$ in the partition $\pi$.

Following Ivanov [15, Section 2], given $\xi \in \mathcal{S P}_{n}$ we define a function on $\mathcal{O P}_{n}$

$$
\widetilde{\phi}^{\xi}= \begin{cases}\phi^{\xi} & \text { if } \xi \in \mathcal{S P}_{n}^{+}, \\ \frac{\phi_{+}^{\xi}+\phi_{-}^{\xi}}{\sqrt{2}}=\sqrt{2} \phi^{\xi} & \text { if } \xi \in \mathcal{S P}_{n}^{-} .\end{cases}
$$

Then the link between such spin characters $\widetilde{\phi}$ and the transition matrix $X$ is provided by the equality

$$
X^{\xi}(\pi)=2^{\frac{\ell(\xi)-\ell(\pi)}{2}} \widetilde{\phi}^{\xi}(\pi)
$$

cf. [15, Proposition 3.3]. 
With these notations (3) can be rewritten as

$$
\mathrm{Ch}_{\pi}^{\text {spin }}(\xi):= \begin{cases}n^{\downarrow k} \frac{X^{\xi}\left(\pi \cup 1^{n-k}\right)}{X^{\xi}\left(1^{n}\right)}=n^{\downarrow k} 2^{\frac{k-\ell(\pi)}{2}} \frac{\widetilde{\phi}^{\xi}\left(\pi \cup 1^{n-k}\right)}{\widetilde{\phi}^{\xi}\left(1^{n}\right)} & \text { if } n \geqslant k, \\ 0 & \text { otherwise. }\end{cases}
$$

\section{SPIN CharaCters in TERMS OF LiNEAR CHARACTERS}

3.1. The main Result 1. We were not able to find this theorem in the literature and we believe it is new. Note, however, that this result is essentially only a reformulation of the classical equality (18) which gives a relation between Schur polynomial $s_{D(\xi)}$ and Schur's $Q$-function $Q_{\xi}$.

We feel that this result is rather unexpected because the spin characters are precisely those that are not obtained by lifting the usual linear characters of the symmetric group; for this reason one should not expect any easy links between the linear and the spin characters.

TheOREM 3.1 (The main result 1: linear in terms of spin). For any $\pi \in \mathcal{O P}$ and $\xi \in \mathcal{S P}$,

$$
\mathrm{Ch}_{\pi}(D(\xi))=\sum_{S \subseteq[\ell(\pi)]} \mathrm{Ch}_{\pi(S)}^{\text {spin }}(\xi) \mathrm{Ch}_{\pi\left(S^{c}\right)}^{\text {spin }}(\xi)
$$

where $\pi(S)=\left(\pi_{i_{1}}, \pi_{i_{2}}, \ldots, \pi_{i_{r}}\right)$ for $S=\left\{i_{1}<i_{2}<\cdots<i_{r}\right\}$ and $S^{c}=[\ell(\pi)] \backslash S$ denotes the complement of $S$.

The proof is presented below in Section 3.2.

Consider the case $\pi \neq \varnothing$. Each summand on the right-hand side of (15) is equal to another summand in which the roles of the set $S$ and its complement $S^{c}$ are interchanged. By grouping such pairs of summands (each such a pair corresponds to a setpartition of $[\ell(\pi)]$ to at most two blocks) and by dividing both sides of (15) by 2 , the above result can be reformulated as the following equality between functions on $\mathcal{S P}$ :

$$
\widetilde{\mathrm{Ch}}_{\pi}=\sum_{\substack{I: \\|I| \leqslant 2}} \prod_{b \in I} \mathrm{Ch}_{\left(\pi_{i}: i \in b\right)}^{\mathrm{spin}},
$$

where the sum runs over set-partitions $I$ of the set $[\ell(\pi)]$ into at most two blocks, and where we denote

$$
\widetilde{\mathrm{Ch}}_{\pi}(\xi):=\frac{1}{2} \mathrm{Ch}_{\pi}(D(\xi))
$$

for $\xi \in \mathcal{S P}$ and $\pi \in \mathcal{O P}$.

EXAMPLE 3.2. Theorem 3.1 implies the following equalities between functions on the set of strict partitions:

$$
\left\{\begin{aligned}
\widetilde{\mathrm{Ch}}_{k_{1}} & =\mathrm{Ch}_{k_{1}}^{\text {spin }} \\
\widetilde{\mathrm{Ch}}_{k_{1}, k_{2}} & =\mathrm{Ch}_{k_{1}, k_{2}}^{\text {spin }}+\mathrm{Ch}_{k_{1}}^{\text {spin }} \mathrm{Ch}_{k_{2}}^{\text {spin }} \\
\widetilde{\mathrm{Ch}}_{k_{1}, k_{2}, k_{3}} & =\mathrm{Ch}_{k_{1}, k_{2}, k_{3}}^{\text {spin }}+\mathrm{Ch}_{k_{1}, k_{2}}^{\text {spin }} \mathrm{Ch}_{k_{3}}^{\text {spin }}+\mathrm{Ch}_{k_{1}, k_{3}}^{\text {spin }} \mathrm{Ch}_{k_{2}}^{\text {spin }}+\mathrm{Ch}_{k_{2}, k_{3}}^{\text {spin }} \mathrm{Ch}_{k_{1}}^{\text {spin }} \\
\vdots &
\end{aligned}\right.
$$

for arbitrary odd integers $k_{1}, k_{2}, \ldots \geqslant 1$. 


\subsection{Proof of Theorem 3.1.}

Proof of Theorem 3.1. We denote by $f^{\lambda}=\chi^{\lambda}\left(1^{|\lambda|}\right)$ the number of standard tableaux of shape $\lambda$. For a strict partition $\xi$ we denote

$$
g^{\xi}=X^{\xi}\left(1^{|\xi|}\right)
$$

which also happens to be the number of shifted standard tableaux with the shape given by the shifted Young diagram $\xi$, see [21, III-8, Example 12].

Recall the symmetric function algebra $\Lambda=\mathbb{Q}\left[p_{1}, p_{2}, p_{3}, \ldots\right]$ and its subalgebra, the algebra of supersymmetric functions $\Gamma=\mathbb{Q}\left[p_{1}, p_{3}, p_{5}, \ldots\right]$, where the $p_{r}$ are Newton's power-sums. Define the algebra homomorphism $\varphi: \Lambda \rightarrow \Gamma$ by

$$
\varphi\left(p_{r}\right)= \begin{cases}2 p_{r} & \text { if } r \text { is odd } \\ 0 & \text { if } r \text { is even }\end{cases}
$$

Then [21, III-8, Example 10] implies that for any strict partition $\xi$ we have

$$
\varphi\left(s_{D(\xi)}\right)=2^{-\ell(\xi)}\left(Q_{\xi}\right)^{2},
$$

where $Q_{\xi}=Q_{\xi}(x ;-1)$ denotes Schur's $Q$-function [21, Section III.8].

Recall the Frobenius formula for Schur functions [21, I. (7.7)]:

$$
s_{\mu}=\sum_{\pi} z_{\pi}^{-1} \chi^{\mu}(\pi) p_{\pi} .
$$

Applying the homomorphism $\varphi$ to this identity with $\mu=D(\xi)$, we obtain

$$
\varphi\left(s_{D(\xi)}\right)=\sum_{\pi \in \mathcal{O} \mathcal{P}_{2 n}} 2^{\ell(\pi)} z_{\pi}^{-1} \chi^{D(\xi)}(\pi) p_{\pi} .
$$

And, recall the Frobenius formula for Schur $Q$-functions [21, III.(7.5) and Example III.8.11(c)]:

$$
Q_{\xi}=\sum_{\nu \in \mathcal{O P}_{n}} 2^{\ell(\nu)} z_{\nu}^{-1} X^{\xi}(\nu) p_{\nu}
$$

Substituting the above two formulas to (18), we have for any $\xi \in \mathcal{S P}_{n}$

$$
\sum_{\pi \in \mathcal{O P}_{2 n}} 2^{\ell(\pi)} z_{\pi}^{-1} \chi^{D(\xi)}(\pi) p_{\pi}=2^{-\ell(\xi)}\left(\sum_{\nu \in \mathcal{O} \mathcal{P}_{n}} 2^{\ell(\nu)} z_{\nu}^{-1} X^{\xi}(\nu) p_{\nu}\right)^{2} .
$$

By comparing the coefficients of $p_{\left(1^{2 n}\right)}=p_{\left(1^{n}\right)} p_{\left(1^{n}\right)}$ in both sides of (19), we find

$$
\frac{f^{D(\xi)}}{(2 n) !}=2^{-\ell(\xi)}\left(\frac{g^{\xi}}{n !}\right)^{2}
$$

For an alternative proof of this identity see [20, Proposition 3.1].

First we assume that $\pi$ is an odd partition which does not have parts equal to 1 , i.e., $m_{1}(\pi)=0$. By comparing the coefficients of $p_{\pi \cup\left(1^{2 n-|\pi|}\right)}$ in both sides of (19) we find

$$
\frac{\chi^{D(\xi)}\left(\pi \cup\left(1^{n-|\pi|}\right)\right)}{z_{\pi \cup\left(1^{n-|\pi|}\right)}}=2^{-\ell(\xi)} \sum_{\substack{\mu^{1}, \mu^{2} \\ \mu^{1} \cup \mu^{2}=\pi}} \frac{X^{\xi}\left(\mu^{1} \cup\left(1^{n-\left|\mu^{1}\right|}\right)\right)}{z_{\mu^{1} \cup\left(1^{n-\mid \mu^{1}}\right)}} \frac{X^{\xi}\left(\mu^{2} \cup\left(1^{n-\left|\mu^{2}\right|}\right)\right)}{z_{\mu^{1} \cup\left(1^{n-\mid \mu^{2}} \mid\right)}} .
$$


By the assumption $m_{1}(\pi)=0$, we have $z_{\pi \cup\left(11^{2 n-|\pi|}\right)}=z_{\pi} \cdot(2 n-|\pi|) !$ and $z_{\mu^{i} \cup\left(1^{n-\left|\mu^{i}\right|}\right)}=$ $z_{\mu^{i}} \cdot\left(n-\left|\mu^{i}\right|\right) !$. Thus, we obtain

$$
\frac{\chi^{D(\xi)}\left(\pi \cup\left(1^{n-|\pi|}\right)\right)}{z_{\pi} \cdot(2 n-|\pi|) !}=2^{-\ell(\xi)} \sum_{\substack{\mu^{1}, \mu^{2} \\ \mu^{1} \cup \mu^{2}=\pi}} \frac{X^{\xi}\left(\mu^{1} \cup\left(1^{n-\left|\mu^{1}\right|}\right)\right)}{z_{\mu^{1}} \cdot\left(n-\left|\mu^{1}\right|\right) !} \frac{X^{\xi}\left(\mu^{2} \cup\left(1^{n-\left|\mu^{2}\right|}\right)\right)}{z_{\mu^{2}} \cdot\left(n-\left|\mu^{2}\right|\right) !} .
$$

Taking the quotient of this and (20), we have

$$
\begin{aligned}
& \frac{1}{z_{\pi}} \frac{(2 n) !}{(2 n-|\pi|) !} \frac{\chi^{D(\xi)}\left(\pi \cup\left(1^{2 n-|\pi|}\right)\right)}{f^{D(\xi)}} \\
& =\sum_{\substack{\mu^{1}, \mu^{2} \\
\mu^{1} \cup \mu^{2}=\pi}} \frac{1}{z_{\mu^{1}} z_{\mu^{2}}} \frac{n !}{\left(n-\left|\mu^{1}\right|\right) !} \frac{X^{\xi}\left(\mu^{1} \cup\left(1^{n-\left|\mu^{1}\right|}\right)\right)}{g^{\xi}} \frac{n !}{\left(n-\left|\mu^{2}\right|\right) !} \frac{X^{\xi}\left(\mu^{2} \cup\left(1^{n-\left|\mu^{2}\right|}\right)\right)}{g^{\xi}},
\end{aligned}
$$

which is equivalent to

$$
\mathrm{Ch}_{\pi}(D(\xi))=\sum_{\substack{\mu^{1}, \mu^{2} \\ \mu^{1} \cup \mu^{2}=\pi}} \frac{z_{\pi}}{z_{\mu^{1}} z_{\mu^{2}}} \mathrm{Ch}_{\mu^{1}}^{\mathrm{spin}}(\xi) \mathrm{Ch}_{\mu^{2}}^{\mathrm{spin}}(\xi)
$$

It is easy to see that this is equivalent to the desired formula. Thus, we completed the proof of the theorem under the assumption $m_{1}(\pi)=0$.

Next we consider a general odd partition $\pi$ and we write it as $\pi=\tilde{\pi} \cup\left(1^{r}\right)$, where $m_{1}(\tilde{\pi})=0$ and $r=m_{1}(\pi)$. Then

$$
\sum_{S \subseteq[\ell(\pi)]} \mathrm{Ch}_{\pi(S)}^{\mathrm{spin}}(\xi) \mathrm{Ch}_{\pi\left(S^{c}\right)}^{\mathrm{spin}}(\xi)=\sum_{T \subseteq[\ell(\tilde{\pi})]} \sum_{s=0}^{r}\left(\begin{array}{l}
r \\
s
\end{array}\right) \mathrm{Ch}_{\tilde{\pi}(T) \cup\left(1^{s}\right)}^{\mathrm{spin}}(\xi) \mathrm{Ch}_{\tilde{\pi}\left(T^{c}\right) \cup\left(1^{r-s}\right)}^{\mathrm{spin}}(\xi),
$$

where $T^{c}=[\ell(\tilde{\pi})] \backslash T$. By virtue of the identity

$$
\mathrm{Ch}_{\nu \cup\left(1^{s}\right)}^{\text {spin }}(\xi)=(n-|\nu|)^{\downarrow s} \mathrm{Ch}_{\nu}^{\text {spin }}(\xi),
$$

we have

$$
\begin{aligned}
(21)=\sum_{T \subseteq[\ell(\tilde{\pi})]} \operatorname{Ch}_{\tilde{\pi}(T)}^{\text {spin }}(\xi) & \operatorname{Ch}_{\tilde{\pi}\left(T^{c}\right)}^{\text {spin }}(\xi) \\
& \times \sum_{s=0}^{r} \frac{r !}{s !(r-s) !} \frac{(n-|\tilde{\pi}(T)|) !}{(n-|\tilde{\pi}(T)|-s) !} \frac{\left(n-\left|\tilde{\pi}\left(T^{c}\right)\right|\right) !}{\left(n-\left|\tilde{\pi}\left(T^{c}\right)\right|-(r-s)\right) !} .
\end{aligned}
$$

Here it is easy to see that

$$
\begin{aligned}
& \sum_{s=0}^{r} \frac{r !}{s !(r-s) !} \frac{(n-|\tilde{\pi}(T)|) !}{(n-|\tilde{\pi}(T)|-s) !} \frac{\left(n-\left|\tilde{\pi}\left(T^{c}\right)\right|\right) !}{\left(n-\left|\tilde{\pi}\left(T^{c}\right)\right|-(r-s)\right) !} \\
& =r ! \sum_{s=0}^{r}\left(\begin{array}{c}
n-|\tilde{\pi}(T)| \\
s
\end{array}\right)\left(\begin{array}{c}
n-\left|\tilde{\pi}\left(T^{c}\right)\right| \\
r-s
\end{array}\right)=r !\left(\begin{array}{c}
2 n-|\tilde{\pi}(T)|-\left|\tilde{\pi}\left(T^{c}\right)\right| \\
r
\end{array}\right)=\frac{(2 n-|\tilde{\pi}|) !}{(2 n-|\tilde{\pi}|-r) !}
\end{aligned}
$$


Thus we have obtained

$$
\begin{aligned}
\sum_{S \subseteq[\ell(\pi)]} \mathrm{Ch}_{\pi(S)}^{\text {spin }}(\xi) \mathrm{Ch}_{\pi\left(S^{c}\right)}^{\text {spin }}(\xi) & =\frac{(2 n-|\tilde{\pi}|) !}{(2 n-|\tilde{\pi}|-r) !} \sum_{S \subseteq[\ell(\tilde{\pi})]} \operatorname{Ch}_{\tilde{\pi}(T)}^{\text {spin }}(\xi) \operatorname{Ch}_{\tilde{\pi}\left(T^{c}\right)}^{\text {spin }}(\xi) \\
& =\frac{(2 n-|\tilde{\pi}|) !}{(2 n-|\tilde{\pi}|-r) !} \mathrm{Ch}_{\tilde{\pi}}(D(\xi)) \\
& =\mathrm{Ch}_{\pi}(D(\xi))
\end{aligned}
$$

which concludes the proof. Here the second equality follows from the previous part of the present proof and the third equality follows from (22).

3.3. The main Result 2. Spin Characters in terms of linear characters. Formulas (17) can be viewed as an upper-triangular system of equations with unknowns $\left(\mathrm{Ch}_{\pi}^{\text {spin }}\right)_{\pi \in \mathcal{O} \mathcal{P}}$. It can be solved, for example

$$
\left\{\begin{aligned}
\mathrm{Ch}_{k_{1}}^{\text {spin }} & =\widetilde{\mathrm{Ch}}_{k_{1}}, \\
\mathrm{Ch}_{k_{1}, k_{2}}^{\text {spin }} & =\widetilde{\mathrm{Ch}}_{k_{1}, k_{2}}-\widetilde{\mathrm{Ch}}_{k_{1}} \widetilde{\mathrm{Ch}}_{k_{2}}, \\
\mathrm{Ch}_{k_{1}, k_{2}, k_{3}}^{\mathrm{spin}} & =\widetilde{\mathrm{Ch}}_{k_{1}, k_{2}, k_{3}} \\
& -\widetilde{\mathrm{Ch}}_{k_{1}, k_{2}} \widetilde{\mathrm{Ch}}_{k_{3}}-\widetilde{\mathrm{Ch}}_{k_{1}, k_{3}} \widetilde{\mathrm{Ch}}_{k_{2}}-\widetilde{\mathrm{Ch}}_{k_{2}, k_{3}} \widetilde{\mathrm{Ch}}_{k_{1}} \\
& +3 \widetilde{\mathrm{Ch}}_{k_{1}} \widetilde{\mathrm{Ch}}_{k_{2}} \widetilde{\mathrm{Ch}}_{k_{3}}, \\
\vdots &
\end{aligned}\right.
$$

The general pattern is given by the following result.

Theorem 3.3 (The main result 2: spin in terms of linear). For any $\pi \in \mathcal{O P}$ such that $\pi \neq \varnothing$ the following equality between functions on the set $\mathcal{S P}$ of strict partitions holds true:

$$
\mathrm{Ch}_{\pi}^{\text {spin }}=\sum_{I}(-1)^{|I|-1}(2|I|-3) ! ! \prod_{b \in I} \widetilde{\mathrm{Ch}}_{\left(\pi_{i}: i \in b\right)},
$$

where the sum runs over all set-partitions of the set $[\ell(\pi)]$ and where we use the convention that $(-1) ! !=1$.

Proof. By singling out the partition $I$ in (16) which consists of exactly one block we may express the spin character $\mathrm{Ch}_{\pi}^{\text {spin }}$ in terms of the linear character $\widetilde{\mathrm{Ch}}_{\pi}$ and spin characters $\mathrm{Ch}_{\pi^{\prime}}^{\text {spin }}$ which correspond to partitions $\pi^{\prime} \in \mathcal{O P}$ with $\ell\left(\pi^{\prime}\right)<\ell(\pi)$ :

$$
\mathrm{Ch}_{\pi}^{\text {spin }}=\widetilde{\mathrm{Ch}}_{\pi}-\sum_{\substack{I:: \\|I|=2}} \prod_{b \in I} \mathrm{Ch}_{\left(\pi_{i}: i \in b\right)}^{\text {spin }} .
$$

By applying this procedure recursively to the spin characters on the right-hand side, we end up with an expression for $\mathrm{Ch}_{\pi}^{\text {spin }}$ as a linear combination (with integer coefficients) of the products of the form

$$
\prod_{b \in I} \widetilde{\mathrm{Ch}}_{\left(\pi_{i}: i \in b\right)}
$$

over set-partitions $I$ of $[\ell(\pi)]$. The remaining difficulty is to determine the exact value of the coefficient of (26) in this linear combination.

The above recursive procedure can be encoded by a tree as follows. Each vertex which is a leaf is labelled by some linear character $\widetilde{\mathrm{Ch}}_{\left(\pi_{i}: i \in c\right)}$ for some non-empty subset $c \subseteq[\ell(\pi)]$ or — to keep the notation light — by the set $c$. Each vertex which 
is not a leaf is labelled by some spin character $\mathrm{Ch}_{\left(\pi_{i}: i \in c\right)}^{\text {spin }}$ for some non-empty subset $c \subseteq[\ell(\pi)]$ or — to keep the notation light — by the set $c$.

Each vertex $c \subseteq[\ell(\pi)]$ which is not a leaf has exactly two children $c_{1}, c_{2} \subseteq[\ell(\pi)]$ which are non-empty, disjoint sets such that $c=c_{1} \sqcup c_{2}$. Thus the labels of all non-leafs are uniquely determined by the labels of the leafs, so we may remove these non-leaf labels. Since we are interested in the coefficient of (26), we require that the set of leaf labels is equal to the set of blocks of $I$ from (26).

The resulting non-ordered trees with the property that each non-leaf vertex has exactly two children are known as total binary partitions; the cardinality of such trees with the prescribed set $I$ of leaf labels is equal to $(2|I|-3)$ !!, cf. [34, Example 5.2.6].

Our recursive procedure involves change of the sign; such a change occurs once for each non-leaf vertex. Thus each total binary tree contributes with multiplicity $(-1)^{|I|-1}$ which concludes the proof.

\section{Proof of spin Stanley Character formula}

Proof of Theorem 1.4. We start with Theorem 3.3 and substitute each normalized linear character

$$
\widetilde{\mathrm{Ch}}_{\left(\pi_{i}: i \in b\right)}(\xi)=\frac{1}{2} \mathrm{Ch}_{\left(\pi_{i}: i \in b\right)}(D(\xi))
$$

which contributes to the right-hand side of (24) by the linear Stanley character formula (6).

We shall discuss in detail the case when $\pi=\left(\pi_{1}, \pi_{2}\right)$ consists of just two parts. We will view $\mathfrak{S}_{\pi_{1}}, \mathfrak{S}_{\pi_{2}}$ and $\mathfrak{S}_{\pi_{1}+\pi_{2}}$ as the groups of permutations of, respectively, the set $\left\{1, \ldots, \pi_{1}\right\},\left\{\pi_{1}+1, \ldots, \pi_{1}+\pi_{2}\right\}$ and $\left\{1, \ldots, \pi_{1}+\pi_{2}\right\}$. In this way we may identify $\mathfrak{S}_{\pi_{1}} \times \mathfrak{S}_{\pi_{2}}$ as a subgroup of $\mathfrak{S}_{\pi_{1}+\pi_{2}}$. As usually, we identify $\left(\pi_{1}\right) \in \mathfrak{S}_{\pi_{1}},\left(\pi_{2}\right) \in$ $\mathfrak{S}_{\pi_{2}}$ with arbitrary permutations with prescribed cycle structures; then $\left(\pi_{1}, \pi_{2}\right):=$ $\left(\left(\pi_{1}\right),\left(\pi_{2}\right)\right) \in \mathfrak{S}_{\pi_{1}} \times \mathfrak{S}_{\pi_{2}} \subseteq \mathfrak{S}_{\pi_{1}+\pi_{2}}$ is also a permutation with appropriate cycle structure. With these notations we have

$$
\begin{aligned}
& \mathrm{Ch}_{\left(\pi_{1}, \pi_{2}\right)}^{\text {spin }}(\xi)=\frac{(-1) ! !}{2} \mathrm{Ch}_{\left(\pi_{1}, \pi_{2}\right)}(D(\xi))-\frac{1 ! !}{2^{2}} \mathrm{Ch}_{\left(\pi_{1}\right)}(D(\xi)) \mathrm{Ch}_{\left(\pi_{2}\right)}(D(\xi)) \\
& =\frac{(-1) ! !}{2} \sum_{\substack{\sigma_{1}, \sigma_{2} \in \mathfrak{S}_{\pi_{1}+\pi_{2}} \\
\sigma_{1} \sigma_{2}=\left(\pi_{1}, \pi_{2}\right)}}(-1)^{\sigma_{1}} N_{\sigma_{1}, \sigma_{2}}(D(\xi)) \\
& -\frac{1 ! !}{2^{2}} \sum_{\substack{\sigma_{1}^{(1)}, \sigma_{2}^{(1)} \in \mathfrak{S}_{\pi_{1}} \\
\sigma_{1}^{(1)} \sigma_{2}^{(1)}=\left(\pi_{1}\right)}}(-1)^{\sigma_{1}^{(1)}} N_{\sigma_{1}^{(1)}, \sigma_{2}^{(1)}}(D(\xi)) \\
& \times \sum_{\substack{\sigma_{1}^{(2)}, \sigma_{2}^{(2)} \in \mathfrak{S}_{\pi_{2}} \\
\sigma_{1}^{(2)} \sigma_{2}^{(2)}=\left(\pi_{2}\right)}}(-1)^{\sigma_{1}^{(2)}} N_{\sigma_{1}^{(2)}, \sigma_{2}^{(2)}}(D(\xi)) \\
& =\frac{(-1) ! !}{2} \sum_{\substack{\sigma_{1}, \sigma_{2} \in \mathfrak{S}_{\pi_{1}+\pi_{2}} \\
\sigma_{1} \sigma_{2}=\left(\pi_{1}, \pi_{2}\right)}}(-1)^{\sigma_{1}} N_{\sigma_{1}, \sigma_{2}}(D(\xi)) \\
& -\frac{1 ! !}{2^{2}} \sum_{\substack{\sigma_{1}, \sigma_{2} \in \mathfrak{S}_{\pi_{1}} \times \mathfrak{S}_{\pi_{2}} \\
\sigma_{1} \sigma_{2}=\left(\pi_{1}, \pi_{2}\right)}}(-1)^{\sigma_{1}} N_{\sigma_{1}, \sigma_{2}}(D(\xi)),
\end{aligned}
$$

where the last equality follows from identification between the pair $\left(\sigma_{i}^{(1)}, \sigma_{i}^{(2)}\right)$ with the corresponding permutation $\sigma_{i} \in \mathfrak{S}_{\pi_{1}} \times \mathfrak{S}_{\pi_{2}}$. 
In general,

$$
\mathrm{Ch}_{\pi}^{\mathrm{spin}}(\xi)=\sum_{\substack{\sigma_{1}, \sigma_{2} \in \mathfrak{S}_{|\pi|} \\ \sigma_{1} \sigma_{2}=\pi}} c_{\sigma_{1}, \sigma_{2}}(-1)^{\sigma_{1}} N_{\sigma_{1}, \sigma_{2}}(D(\xi))
$$

for some combinatorial factor $c_{\sigma_{1}, \sigma_{2}}$. For example, in the special case $\pi=\left(\pi_{1}, \pi_{2}\right)$ which consists of two parts, (27) implies that

$$
c_{\sigma_{1}, \sigma_{2}}= \begin{cases}\frac{(-1) ! !}{2}-\frac{1 ! !}{2^{2}} & \text { if } \sigma_{1}, \sigma_{2} \in \mathfrak{S}_{\pi_{1}} \times \mathfrak{S}_{\pi_{2}}, \\ \frac{(-1) ! !}{2} & \text { otherwise. }\end{cases}
$$

We claim that in the general case the value of the constant $c_{\sigma_{1}, \sigma_{2}}$ is equal to

$$
C_{m}:=c_{\sigma_{1}, \sigma_{2}}=(-1) \sum_{p}\left\{\begin{array}{c}
m \\
p
\end{array}\right\}\left(-\frac{1}{2}\right)^{p}(2 p-3) ! !,
$$

where $m=\left|\sigma_{1} \vee \sigma_{2}\right|$ is the number of orbits in the set $[|\pi|]$ under the action of the group $\left\langle\sigma_{1}, \sigma_{2}\right\rangle$ generated by $\sigma_{1}, \sigma_{2}$, and $\left\{\begin{array}{c}m \\ k\end{array}\right\}$ denotes Stirling numbers of the second kind. Indeed, the set-partition $I$ (over which we sum in (24)) can be identified with a set-partition on the set of the cycles of the permutation $\pi \in \mathfrak{S}_{|\pi|}$. With this in mind we see that to $c_{\sigma_{1}, \sigma_{2}}$ contribute only these set-partitions $I$ on the right-hand side of (24) for which $I$ is bigger than the set-partition given by the orbits of $\left\langle\sigma_{1}, \sigma_{2}\right\rangle$. The collection of such set-partitions can be identified with the collection of set-partitions of an $m$-element set (i.e. the set of orbits of $\left\langle\sigma_{1}, \sigma_{2}\right\rangle$ ). For a fixed value $p:=|I|$ of the number of the blocks there are clearly $\left\{\begin{array}{c}m \\ p\end{array}\right\}$ choices of $I$; each contributes to $c_{\sigma_{1}, \sigma_{2}}$ with the multiplicity

$$
(-1)^{p-1}(2 p-3) ! ! \frac{1}{2^{p}}
$$

this concludes the proof of (29).

The sum in (29) can be probably calculated by a clever trick which we, regretfully, failed to find. From our perspective the exact form of the right-hand side of (29) is not important; in the following we will make use only of the observation that $c_{\sigma_{1}, \sigma_{2}}=C_{m}$ depends only on the number of the orbits of $\left\langle\sigma_{1}, \sigma_{2}\right\rangle$.

In order to evaluate $C_{m}$ we shall consider (28) in the special case of $\pi=1^{m}$. In this case $\sigma_{2}=\sigma_{1}^{-1}$; we denote by $l=\left|C\left(\sigma_{1}\right)\right|$ the number of cycles of $\sigma_{1}$. It follows that

$$
\mathrm{Ch}_{1^{m}}^{\mathrm{spin}}(\xi)=n^{\downarrow m}=\sum_{l}\left[\begin{array}{c}
m \\
l
\end{array}\right] C_{l}(-1)^{m-l}(2 n)^{l},
$$

where $n=|\xi|$ and $\left[\begin{array}{c}m \\ l\end{array}\right]$ denotes Stirling number of the first kind. Both sides of the equality are polynomials in the variable $n$; by comparing the leading coefficients (which corresponds to setting $l=m$ ) we conclude that

$$
C_{m}=\frac{1}{2^{m}}
$$

By substituting this value into (28) we conclude the proof.

\section{Proof of Theorem 1.7}

Maps which we consider come in two distinct flavours: non-oriented maps versus oriented maps. We review their difference in the following. 
5.1. Non-ORIENTED MAPS, REVISITED. We keep notations from Sections 1.5.1 and 1.5.2. The polygons from the collection $\mathcal{P}$ have labelled edges. These labels can be erased and we would be still able to recover them if we preserve the following information:

- we paint the polygons from the collection $\mathcal{P}$ with distinct colors;

- on each polygon from $\mathcal{P}$ we select some white vertex ("root");

- on each polygon from $\mathcal{P}$ we select one of the two edges adjacent to the root ( "direction of rotation ${ }^{(2)}$ ").

The roots and the directions of rotation were indicated on Figure 3 by arrows and harpoons respectively.

We recall that the maps which we consider are obtained by gluing the polygons with labelled edges. As a result, each edge of the map carries two labels; one on each side of the edge, see Figure 4 . We can erase these labels and still be able to recover them if we preserve the following information:

- we paint each face of the map with the colour of the corresponding polygon from $\mathcal{P}$ (so that we know the correspondence between the faces of the map and the polygons);

- on each face of the map we decorate the white corner ("root") which corresponds to the root of the corresponding polygon from $\mathcal{P}$;

- on each face we decorate one of the two edges adjacent to the root which corresponds to the direction of rotation of the corresponding polygon from $\mathcal{P}$.

The roots and the directions of rotation are indicated on Figure 4 by arrows and harpoons respectively.

5.2. ORIEnTED MAPS. We say that a map is oriented if the surface $\Sigma$ is orientable and on each connected component some orientation is selected, see Figure 6 .

Summation over oriented maps with a specified face-type $\pi$ should be understood as follows: we start by fixing an appropriate collection $\mathcal{P}$ of polygons with labelled edges, bicolored vertices, and with face-type $\pi$. Additionally, on each polygon we choose some orientation ("which direction of rotation should be understood as clockwise"), see Figure 5. Then we consider only these perfect matchings on the set of all edges, which result with a glueing of the polygons with the property that the originally selected orientations on the polygons are consistent when one crosses an edge. We sum over the resulting oriented map.

5.3. LABELS ON ORIENTED MAPS. In the context of the oriented maps it is more convenient to label only some edges of the oriented polygons from $\mathcal{P}$; more specifcially we label only these edges which - if one traverses the edges in the clockwise cyclic order — start with a white vertex, see Figure 5. The labelling can be encoded by a permutation $\pi$, the cycles of which correspond to the cyclic order of the labels around the polygons (in the clockwise direction), see Figure 5.

With this convention, the orientations of the polygons are consistent in the map obtained by glueing the edges of $\mathcal{P}$ if and only if in each pair of glued edges exactly one carries a label, see Figure 6.

The structure of such an oriented map can be uniquely recovered from the pair of permutations $\sigma_{1}, \sigma_{2}$, where the cycles of $\sigma_{1}$ encode the counterclockwise cyclic order around the white vertices while the cycles of $\sigma_{2}$ encode the counterclockwise cyclic

\footnotetext{
${ }^{(2)}$ Even though the name orientation would sound more appropriate here, we decided to reserve the latter word for the context of oriented maps.
} 


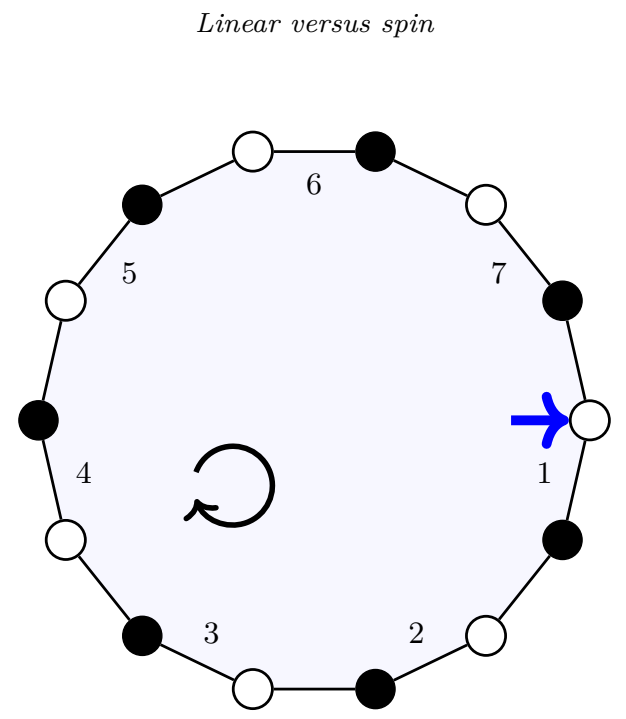

Figure 5. A collection of oriented polygons of face type (7). The labeling of the edges corresponds to the permutation $\pi=$ $(1,2,3,4,5,6,7) \in \mathfrak{S}_{7}$. The root is indicated by the thick blue arrow. The orientation is indicated by the circular arrow.

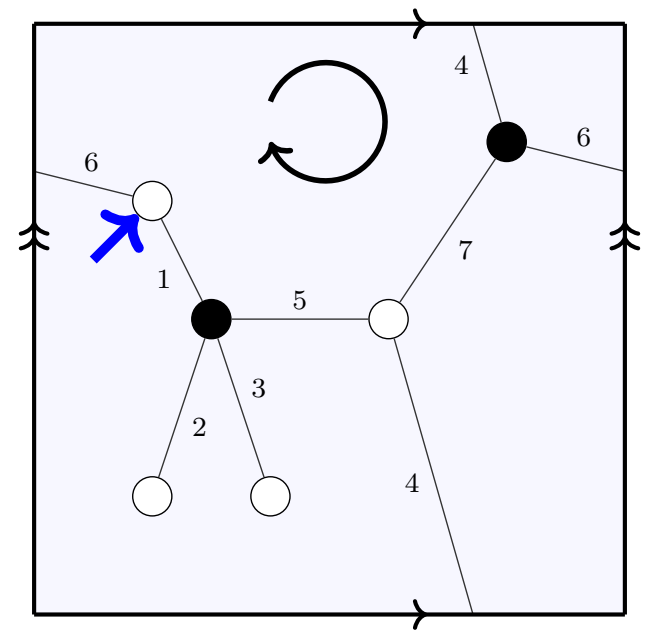

FiguRE 6. Example of an oriented map. The map is drawn on a torus: the left side of the square should be glued to the right side, as well as bottom to top, as indicated by the arrows. The thick blue arrow indicates the root of the face. The orientation is indicated by the circular arrow. This map was created by glueing the edges of the oriented collection of polygons from Figure 5 .

order around the black vertices. For example, for the map from Figure 6 we have

$$
\begin{aligned}
& \sigma_{1}=(1,6)(2)(3)(4,7,5), \\
& \sigma_{2}=(1,2,3,5)(4,7,6) .
\end{aligned}
$$

It is easy to see that the product

$$
\pi=\sigma_{1} \sigma_{2}
$$


is the aforementioned permutation $\pi$ which gives the clockwise order of the labels on the polygons from $\mathcal{P}$. In our example

$$
\pi=\sigma_{1} \sigma_{2}=(1,2,3,4,5,6,7)
$$

see Figure 5.

With this in mind, it is easy to see that, for a given integer partition $\pi$, the summation over oriented maps with face-type $\pi$ is equivalent to fixing some permutation $\pi \in \mathfrak{S}_{|\pi|}$ with the cycle decomposition given by the partition $\pi$, and considering all solutions to the equation

$$
\left\{\left(\sigma_{1}, \sigma_{2}\right): \sigma_{1}, \sigma_{2} \in \mathfrak{S}_{|\pi|}, \quad \sigma_{1} \sigma_{2}=\pi\right\} .
$$

5.4. Proof of Theorem 1.7. Our proof will be based on a simple double counting argument.

Proof of Theorem 1.7. In the light of the discussion from Section 5.3, the right-hand side of (7) can be interpreted as a sum over oriented maps with face-type $\pi$.

The oriented maps which we consider have labelled edges. We can remove these labels and still be able to recover them if we preserve the following information:

- we paint each face of the map with the colour of the corresponding polygon from $\mathcal{P}$;

- on each face of the map we decorate the white corner ("root") which corresponds to the root of the corresponding polygon from $\mathcal{P}$;

- on each connected component of the map we indicate the orientation.

There are $2^{\ell(\pi)}$ ways to choose direction of the rotation on each face a map (in the spirit of Section 5.1). It follows from (7) that

$$
\mathrm{Ch}_{\pi}^{\text {spin }}(\xi)=\sum_{M} \frac{1}{2^{c(M)}} \frac{1}{2^{\ell(\pi)}}(-1)^{|\pi|-\left|\mathcal{V}_{\diamond}(M)\right|} N_{M}(D(\xi)),
$$

where the sum runs over oriented maps with coloured faces, with each face having a decorated white corner, and with each face having some selected direction of rotation. Above, $c(M)$ denotes the number of connected components of $M$; with notations of (7) we clearly have $c(M)=\left|\left\langle\sigma_{1}, \sigma_{2}\right\rangle\right|$.

Let us remove the information about the orientation. In the light of Section 5.1, the resulting object is a non-oriented map which happens to be orientable. Clearly, for each such a non-oriented but orientable map there are $2^{c(M)}$ choices for the orientation on each connected component of $M$. It follows that

$$
\mathrm{Ch}_{\pi}^{\mathrm{spin}}(\xi)=\sum_{M} \frac{1}{2^{\ell(\pi)}}(-1)^{|\pi|-\left|\mathcal{V}_{\circ}(M)\right|} N_{M}(D(\xi)),
$$

where the sum runs over non-oriented but orientable maps with face-type $\pi$, as required.

\section{Multirectangular coordinates, Spin Stanley polynomials}

Our ultimate goal is to prove Theorems 1.8 and 1.9 which provide the link between (the filtration on) the linear Kerov-Olshanski algebra $\mathbb{A}$ and its spin counterpart $\Gamma$; we will do this in Section 8.

We start in this section by preparing the tools: multirectangular coordinates and Stanley polynomials. 


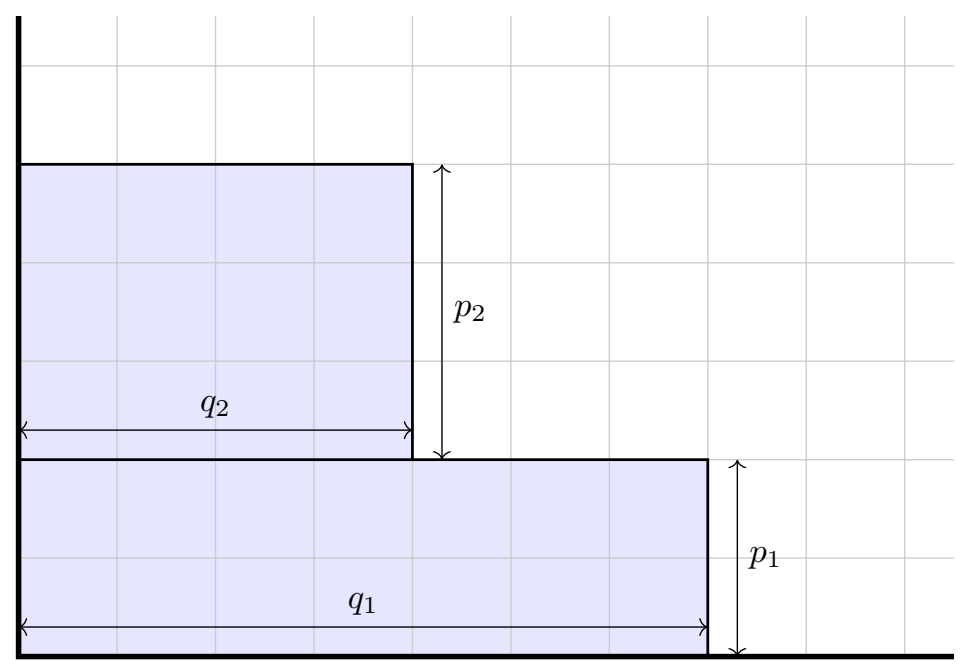

FigURE 7. Multirectangular coordinates for partitions.

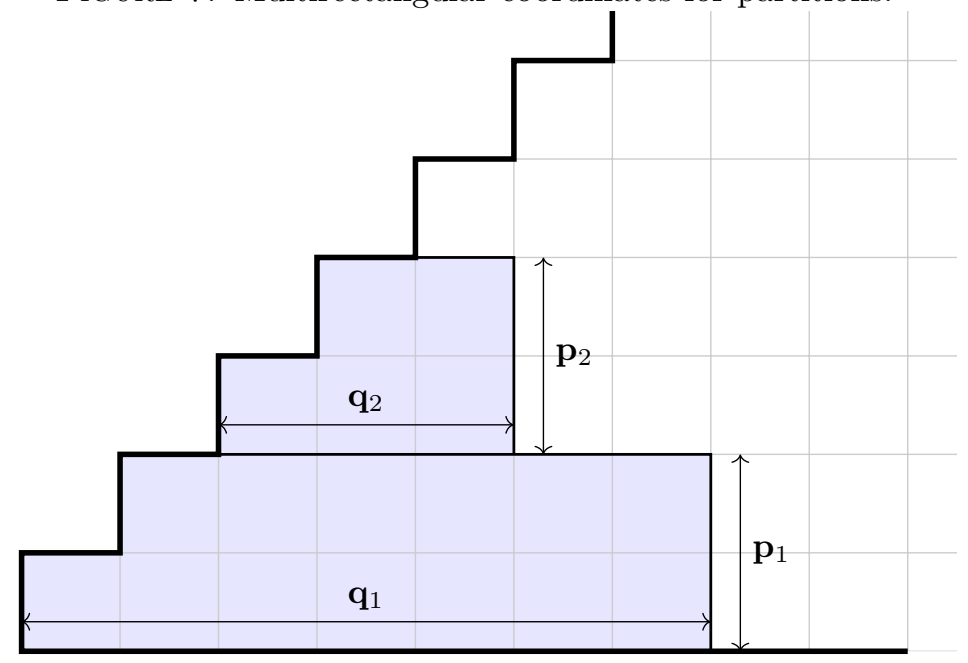

FIGURE 8. Multirectangular coordinates for strict partitions.

6.1. Multirectangular coordinates. Following Stanley [35], for tuples of integers $P=\left(p_{1}, \ldots, p_{l}\right), Q=\left(q_{1}, \ldots, q_{l}\right)$ such that $p_{1}, \ldots, p_{l} \geqslant 0$ and $q_{1} \geqslant \cdots \geqslant q_{l} \geqslant 0$ we consider the corresponding multirectangular partition $P \times Q$, cf. Figure 7 .

De Stavola [5] adapted this notion to strict partitions: for tuples of integers $\mathbf{P}=$ $\left(\mathbf{p}_{1}, \ldots, \mathbf{p}_{l}\right), \mathbf{Q}=\left(\mathbf{q}_{1}, \ldots, \mathbf{q}_{l}\right)$ such that $\mathbf{p}_{1}, \ldots, \mathbf{p}_{l} \geqslant 0$ and $\mathbf{q}_{2} \leqslant \mathbf{q}_{1}-\mathbf{p}_{1}, \mathbf{q}_{3} \leqslant$ $\mathbf{q}_{2}-\mathbf{p}_{2}, \ldots, \mathbf{q}_{l} \leqslant \mathbf{q}_{l-1}-\mathbf{p}_{l-1}, 0 \leqslant \mathbf{q}_{l}-\mathbf{p}_{l}$ he considered a multirectangular strict partition $\mathbf{P} \otimes \mathbf{Q}$, cf. Figure 8; note that in the original work of De Stavola both the multirectangular partition as well as the multirectangular strict partition were denoted by the same symbol $x$.

The following result gives a link between the shifted multirectangular coordinates of a given strict partition $\xi \in \mathcal{S P}$ and the multirectangular coordinates of its double $D(\xi)$. 
LEMMA 6.1. For each $l \geqslant 1$ there exist polynomials

$$
p_{1}, \ldots, p_{l}, q_{1}, \ldots, q_{l} \in \mathbb{Z}\left[\mathbf{p}_{1}, \ldots, \mathbf{p}_{l}, \mathbf{q}_{1}, \ldots, \mathbf{q}_{l}\right] \text { of degree } 1
$$

with the property that

$$
D(\mathbf{P} \otimes \mathbf{Q})=P \times Q
$$

Proof. The desired polynomials are given by

$$
\left\{\begin{array}{rlrl}
p_{1} & =\mathbf{p}_{1}, & q_{1} & =\mathbf{q}_{1}+1, \\
p_{2} & =\mathbf{p}_{2}, & q_{2} & =\mathbf{p}_{1}+\mathbf{q}_{2}+1, \\
\vdots & \vdots & \\
p_{l} & =\mathbf{p}_{l}, & q_{l} & =\mathbf{p}_{1}+\cdots+\mathbf{p}_{l-1}+\mathbf{q}_{l}+1, \\
p_{l+1} & =\mathbf{q}_{l}-\mathbf{p}_{l}, & q_{l+1} & =\mathbf{p}_{1}+\cdots+\mathbf{p}_{l}, \\
p_{l+2} & =\mathbf{q}_{l-1}-\left(\mathbf{q}_{l}+\mathbf{p}_{l-1}\right), & q_{l+2} & =\mathbf{p}_{1}+\cdots+\mathbf{p}_{l-1}, \\
\vdots & & \vdots & \\
p_{2 l} & =\mathbf{q}_{1}-\left(\mathbf{q}_{2}+\mathbf{p}_{1}\right), & q_{2 l} & =\mathbf{p}_{1} .
\end{array}\right.
$$

6.2. Stanley polynomials. Multirectangular coordinates $P, Q$ provide a convenient way of parametrizing partitions; the fact that a partition can be represented in several ways with such coordinates is not relevant.

In particular, for a function $F \in \mathbb{A}$ it is convenient to consider a map

$$
(P, Q) \mapsto F(P \times Q)
$$

which - as it turns out - can be identified with a unique polynomial in the multirectangular coordinates. This polynomial, which is referred to as Stanley polynomial [35], is a convenient tool for studying some enumerative and asymptotic problems of the representation theory of the symmetric groups [7].

The explicit form of this Stanley polynomial $\mathrm{Ch}_{\pi}(P \times Q)$ in the special case when $F=\mathrm{Ch}_{\pi}$ is the normalized character was conjectured by Stanley [36] and proved by Féray [10], see also [11]; we will refer to this result as Stanley character formula (it is just a reformuation of Theorem 1.3).

De Stavola [5] initiated investigation of the analogous coordinates for a function $F \in \Gamma$ and he asked for a closed formula for $\mathrm{Ch}_{\pi}^{\text {spin }}(\mathbf{P} \otimes \mathbf{Q})$.

6.3. Closed formula for spin Stanley polynomial. Multirectangular coordinates are very convenient in the context of Stanley character formula. More specifically, it is easy to see that

$$
N_{\sigma_{1}, \sigma_{2}}(P \times Q)=\sum_{\kappa_{2}} \prod_{c_{1} \in C\left(\sigma_{1}\right)} q_{\kappa_{1}\left(c_{1}\right)} \prod_{c_{2} \in C\left(\sigma_{2}\right)} p_{\kappa_{2}\left(c_{2}\right)},
$$

where the sum runs over all functions $\kappa_{2}: C\left(\sigma_{2}\right) \rightarrow[l]$ and the function $\kappa_{1}: C\left(\sigma_{1}\right) \rightarrow$ $[l]$ is defined by

$$
\kappa_{1}\left(c_{1}\right)=\max \left\{\kappa_{2}\left(c_{2}\right): c_{2} \in C\left(\sigma_{2}\right) \text { and } c_{1} \cap c_{2} \neq \varnothing\right\} \quad \text { for } c_{1} \in C\left(\sigma_{1}\right) .
$$

The explicit form of the Stanley polynomial $\mathrm{Ch}_{\pi}^{\text {spin }}(\mathbf{P} \otimes \mathbf{Q})$ for $\pi \in \mathcal{O P}$ can be found by combining Theorem 1.4 applied to $\xi=\mathbf{P} \otimes \mathbf{Q}$ and $P \times Q=D(\mathbf{P} \otimes \mathbf{Q})$ with Lemma 6.1. In order to prove the uniqueness of this polynomial one can adapt the corresponding part of the proof of [8, Lemma 2.4]. 


\section{Asymptotics of Spin Stanley polynomials}

Recall that our ultimate goal is to prove Theorems 1.8 and 1.9 which provide the link between (the filtration on) the linear Kerov-Olshanski algebra $\mathbb{A}$ and its spin counterpart $\Gamma$.

As an intermediate step we present in this section the link between the filtration on the (linear or spin) Kerov-Olshanski algebra and the degrees of Stanley polynomials (Propositions 7.2 and 7.4) which might be of independent interest.

\subsection{Filtration vs Stanley polynomials: the linear Case.}

LEMma 7.1. For arbitrary integer partition $\pi$ the corresponding linear Stanley polynomial $\mathrm{Ch}_{\pi}(P \times Q)$ is of degree $|\pi|+\ell(\pi)$.

Its homogeneous top-degree part is given by

$$
\left(\mathrm{Ch}_{\pi}\right)^{\text {top }}(P \times Q):=\sum_{\substack{\sigma_{1}, \sigma_{2} \in \mathfrak{S}_{k} \\ \sigma_{1} \sigma_{2}=\pi \\\left|C\left(\sigma_{1}\right)\right|+\left|C\left(\sigma_{2}\right)\right|=|\pi|+\ell(\pi)}}(-1)^{\sigma_{1}} N_{\sigma_{1}, \sigma_{2}}(P \times Q) .
$$

Proof. Our strategy is to investigate the summands on the right-hand side of the linear Stanley character formula (6) . Note that (32) implies that

$$
N_{\sigma_{1}, \sigma_{2}}(P \times Q) \in \mathbb{Z}\left[p_{1}, \ldots, p_{l}, q_{1}, \ldots, q_{l}\right]
$$

is a homogeneous polynomial of degree $\left|C\left(\sigma_{1}\right)\right|+\left|C\left(\sigma_{2}\right)\right|$.

For a permutation $\pi \in \mathfrak{S}_{k}$ we denote by $\|\pi\|:=k-|C(\pi)|$ the minimal number of factors necessary to write $\pi$ as a product of transpositions. Triangle inequality for $\pi=\sigma_{1} \sigma_{2}$ implies that

$$
\left|C\left(\sigma_{1}\right)\right|+\left|C\left(\sigma_{2}\right)\right|=2 k-\left(\left\|\sigma_{1} \mid+\right\| \sigma_{2} \|\right) \leqslant 2 k-\|\pi\|=|\pi|+\ell(\pi) .
$$

It follows therefore that

$$
\mathrm{Ch}_{\pi}(P \times Q)
$$

is indeed a polynomial of degree bounded from above by $|\pi|+\ell(\pi)$, as required.

In order to find the homogeneous top-degree part of this polynomial it is enough to restrict the summation in (7) to the pairs $\sigma_{1}, \sigma_{2}$ for which (35) becomes equality.

Proposition 7.2. Let $F \in \mathbb{A}$ and $k \geqslant 0$ be an integer.

Then $F \in \mathcal{F}_{k}$ if and only if for each integer $l \geqslant 1$

$$
F(P \times Q) \in \mathbb{C}\left[p_{1}, \ldots, p_{l}, q_{1}, \ldots, q_{l}\right]
$$

is a polynomial of degree at most $k$.

Proof. The vector space $\mathcal{F}_{k}$ is a linear span of certain normalized characters; for this reason it is enough to consider $F=\mathrm{Ch}_{\pi} \in \mathcal{F}_{k}$ with $\pi$ as in (11). Application of Lemma 7.1 concludes the proof of the implication in one direction.

We shall prove the opposite implication. Let $m \geqslant 0$ be the minimal integer with the property that $F \in \mathcal{F}_{m}$; our goal is to show that $m \leqslant k$. Suppose that this is not the case and $m>k$. We consider the map

$$
\Psi_{m}:\left.\mathcal{F}_{m} \ni G \mapsto([\text { homogeneous part of degree } m] G(P \times Q))\right|_{p_{1}=\cdots=p_{l}=1}
$$

which selects the top-degree homogeneous part of Stanley's polynomial and afterwards substitutes $p_{1}=\cdots=p_{l}=1$. We will show that $\operatorname{ker} \Psi_{m}=\mathcal{F}_{m-1}$, provided $l \geqslant m$; this would conclude the proof because $m>k$ implies that $F \in \operatorname{ker} \Psi_{m}=\mathcal{F}_{m-1}$ which contradicts the minimality of $m$. 
Clearly the quotient space $\mathcal{F}_{m} / \mathcal{F}_{m-1}$ is a linear span of

$$
\left(\mathrm{Ch}_{\pi}+\mathcal{F}_{m-1}:|\pi|+\ell(\pi)=m\right) .
$$

The map $\Psi_{m}$ maps the family (38) to

$$
\left(\Psi_{m}\left(\mathrm{Ch}_{\pi}\right):|\pi|+\ell(\pi)=m\right) ;
$$

for our purposes of proving $\operatorname{ker} \Psi_{m}=\mathcal{F}_{m-1}$ it is enough to show that (39) is a family of linearly independent vectors. We shall do it in the following.

In order to find the top-degree part of the polynomial

$$
\Psi_{m}\left(\mathrm{Ch}_{\pi}\right) \in \mathbb{Z}\left[q_{1}, \ldots, q_{l}\right]
$$

we combine (33) with (32). This top-degree part corresponds to these summands in (33) for which $\left|C\left(\sigma_{1}\right)\right|$ takes the maximal possible value. This is clearly the summand for $\sigma_{1}=\mathrm{id} \in \mathfrak{S}_{|\pi|}$ and $\sigma_{2}=\pi \in \mathfrak{S}_{|\pi|}$. In this way we proved that (40) is a polynomial of degree $|\pi|$ and its homogeneous part of this maximal degree is equal to the power-sum symmetric function

$$
p_{\pi}\left(q_{1}, \ldots, q_{l}\right)
$$

Suppose that

$$
\sum_{|\pi|+\ell(\pi)=m} a_{\pi} \Psi_{m}\left(\mathrm{Ch}_{\pi}\right)=0
$$

for some coefficients $a_{\pi} \in \mathbb{C}$ which are not all equal to zero and denote $n:=\max \{|\pi|$ : $\left.a_{\pi} \neq 0\right\}$. We consider the homogeneous part of the polynomial on the left-hand side of (42) of degree $n$; by maximality of $n$, only partitions $\pi$ with $|\pi|=n$ contribute, and each such a partition contributes with the power-sum symmetric function (41). Since power-sum symmetric polynomials in $l$ variables of degree $n \leqslant m \leqslant l$ are linearly independent, it follows that $a_{\pi}=0$ for $|\pi|=n$ which contradicts the definition of $n$. This concludes the proof of the equality $\operatorname{ker} \Psi_{m}=\mathcal{F}_{m-1}$.

This result has a spin counterpart (Proposition 7.4), however adapting the above proof to the spin setup requires some preparations which we present below.

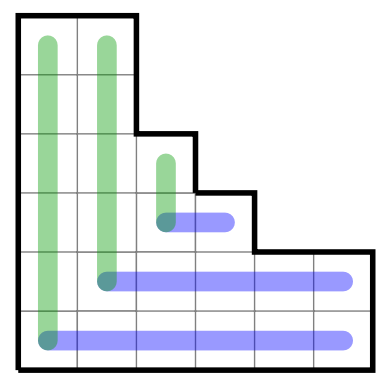

Figure 9. The overlap double $D_{\text {over }}(\xi)=(6,6,4,3,2,2)$ of the strict partition $\xi=(6,5,2)$ from Figure 1 .

7.2. Overlap DOUble of a STRICT PARTItion. For a strict partition $\xi \in \mathcal{S P}$ we consider its overlap double $D_{\text {over }}(\xi) \in \mathcal{P}$ which is obtained by combining the shifted Young diagram $\xi$ and its transpose so that they overlap along the diagonal boxes, cf. Figure 9. 
Let $p_{1}, \ldots, p_{l}, q_{1}, \ldots, q_{l} \in \mathbb{Z}\left[\mathbf{p}_{1}, \ldots, \mathbf{p}_{l}, \mathbf{q}_{1}, \ldots, \mathbf{q}_{l}\right]$ be the homogeneous part of degree 1 of the polynomials (30); in other words

$$
\left\{\begin{aligned}
p_{1} & =\mathbf{p}_{1}, & q_{1} & =\mathbf{q}_{1}, \\
p_{2} & =\mathbf{p}_{2}, & q_{2} & =\mathbf{p}_{1}+\mathbf{q}_{2}, \\
& \vdots & & \\
p_{l} & =\mathbf{p}_{l}, & q_{l} & =\mathbf{p}_{1}+\cdots+\mathbf{p}_{l-1}+\mathbf{q}_{l}, \\
p_{l+1} & =\mathbf{q}_{l}-\mathbf{p}_{l}, & q_{l+1} & =\mathbf{p}_{1}+\cdots+\mathbf{p}_{l}, \\
p_{l+2} & =\mathbf{q}_{l-1}-\left(\mathbf{q}_{l}+\mathbf{p}_{l-1}\right), & q_{l+2} & =\mathbf{p}_{1}+\cdots+\mathbf{p}_{l-1}, \\
\vdots & & \vdots & \\
p_{2 l} & =\mathbf{q}_{1}-\left(\mathbf{q}_{2}+\mathbf{p}_{1}\right), & q_{2 l} & =\mathbf{p}_{1} .
\end{aligned}\right.
$$

It is easy to check that with this choice

$$
D_{\text {over }}(\mathbf{P} \otimes \mathbf{Q})=P \times Q .
$$

7.3. Top-degree Part of SPin Stanley polynomials. Lemma 7.1 has the following spin counterpart.

LEMma 7.3. For arbitrary $\pi \in \mathcal{O P}_{k}$ the corresponding spin Stanley polynomial $\mathrm{Ch}_{\pi}^{\text {spin }}(\mathbf{P} \otimes \mathbf{Q})$ is of degree $|\pi|+\ell(\pi)$. Its homogeneous top-degree part is given by

$$
\begin{aligned}
\left(\mathrm{Ch}_{\pi}^{\text {spin }}\right)^{\text {top }} & (\mathbf{P} \otimes \mathbf{Q}) \\
& :=\sum_{\substack{\sigma_{1}, \sigma_{2} \in \mathfrak{S}_{k} \\
\sigma, \sigma_{2}=\pi \\
\left|C\left(\sigma_{1}\right)\right|+\left|C\left(\sigma_{2}\right)\right|=|\pi|+\ell(\pi)}} \frac{1}{2^{\left|\sigma_{1} \vee \sigma_{2}\right|}}(-1)^{\sigma_{1}} N_{\sigma_{1}, \sigma_{2}}\left(D_{\text {over }}(\mathbf{P} \otimes \mathbf{Q})\right) .
\end{aligned}
$$

Proof. We revisit the proof of Lemma 7.1 but this time we consider the spin Stanley formula (7). Lemma 6.1 implies therefore that

$$
N_{\sigma_{1}, \sigma_{2}}(D(\mathbf{P} \otimes \mathbf{Q})) \in \mathbb{Z}\left[\mathbf{p}_{1}, \ldots, \mathbf{p}_{l}, \mathbf{q}_{1}, \ldots, \mathbf{q}_{l}\right]
$$

is an inhomogeneous polynomial of the degree $\left|C\left(\sigma_{1}\right)\right|+\left|C\left(\sigma_{2}\right)\right|$, obtained from (34) by the substitution (30).

The homogeneous part of (46) of this top degree can therefore be obtained from (34) by the homogeneous substitution (43). Equation (44) implies therefore that this homogeneous part is equal to

$$
N_{\sigma_{1}, \sigma_{2}}\left(D_{\text {over }}(\mathbf{P} \otimes \mathbf{Q})\right) \in \mathbb{Z}\left[\mathbf{p}_{1}, \ldots, \mathbf{p}_{l}, \mathbf{q}_{1}, \ldots, \mathbf{q}_{l}\right] .
$$

Inequality (35) implies therefore that

$$
\mathrm{Ch}_{\pi}^{\mathrm{spin}}(\mathbf{P} \otimes \mathbf{Q})
$$

is indeed a polynomial of degree bounded from above by $|\pi|+\ell(\pi)$, as required.

In order to find the homogeneous top-degree part of this polynomial it is enough to: (a) restrict the summation in (7) to pairs $\sigma_{1}, \sigma_{2}$ for which (35) becomes equality and then (b) to consider the homogeneous part (47) of the surviving summands. This concludes the proof. 


\subsection{Filtration vs Stanley polynomials: the spin Case.}

Proposition 7.4. Let $F \in \Gamma$ and $k \geqslant 0$ be an integer.

Then $F \in \mathcal{G}_{k}$ if and only if for each integer $l \geqslant 1$

$$
F(\mathbf{P} \otimes \mathbf{Q}) \in \mathbb{C}\left[\mathbf{p}_{1}, \ldots, \mathbf{p}_{l}, \mathbf{q}_{1}, \ldots, \mathbf{q}_{l}\right]
$$

is a polynomial of degree at most $k$.

Proof. We revisit the proof of Proposition 7.2 and review the necessary changes.

We replace each occurrence of $\mathcal{F}_{k}$ by $\mathcal{G}_{k}$ and each linear character $\mathrm{Ch}_{\pi}$ with $\pi \in \mathcal{P}$ by its spin counterpart $\mathrm{Ch}_{\pi}^{\text {spin }}$ with $\pi \in \mathcal{O P}$. Reference to Lemma 7.1 should be replaced by Lemma 7.3.

Instead of $\Psi_{m}$ we consider the map

$$
\Psi_{m}^{\text {spin }}:\left.\mathcal{G}_{m} \ni G \mapsto([\text { homogeneous part of degree } m] G(\mathbf{P} \otimes \mathbf{Q}))\right|_{\mathbf{p}_{1}=\cdots=\mathbf{p}_{l}=1}
$$

which selects the top-degree homogeneous part of spin Stanley's polynomial and afterwards substitutes $\mathbf{p}_{1}=\cdots=\mathbf{p}_{l}=1$.

The only part which requires some changes in the spin context is the proof that if $m=|\pi|+\ell(\pi)$ then $\Psi_{m}^{\text {spin }}\left(\mathrm{Ch}_{\pi}^{\text {spin }}\right) \in \mathbb{Q}\left[\mathbf{q}_{1}, \ldots, \mathbf{q}_{l}\right]$ is a polynomial of degree $|\pi|$ and its homogeneous part of this maximal degree is equal to the power-sum symmetric function $p_{\pi}\left(\mathbf{q}_{1}, \ldots, \mathbf{q}_{l}\right)$. We present this proof below.

Let us fix some $\sigma_{1}, \sigma_{2} \in \mathfrak{S}_{|\pi|}$ which contribute to (45). The colorings which contribute to $N_{\sigma_{1}, \sigma_{2}}(\lambda)$ with

$$
\lambda=D_{\text {over }}((1, \ldots, 1) \otimes \mathbf{Q})=D_{\text {over }}\left(\mathbf{q}_{1}, \ldots, \mathbf{q}_{l}\right)
$$

can be enumerated by the following algorithm. Firstly, we select some set of cycles of $\sigma_{1}$ and some set of cycles of $\sigma_{2}$ (we will call the cycles which belong to them special cycles). Secondly, we assign to these special cycles the values of $f_{1}$ and $f_{2}$ from the set $[l]$ in an arbitrary way. The above two steps do not depend on the variables $\mathbf{q}_{1}, \ldots, \mathbf{q}_{l}$, but they do depend on $l$, the number of the variables.

In the third step we associate to all non-special cycles the values of $f_{1}$ and $f_{2}$ from the set $\{l+1, l+2, \ldots\}$ in such a way that $f=\left(f_{1}, f_{2}\right)$ is a colouring of $\left(\sigma_{1}, \sigma_{2}\right)$ which is compatible with $\lambda$. Without loss of generality we may assume that whenever two cycles $c_{1} \in C\left(\sigma_{1}\right), c_{2} \in C\left(\sigma_{2}\right)$ intersect, at least one of them is special; otherwise the column $f_{1}\left(c_{1}\right)$ and the row $f_{2}\left(c_{2}\right)$ would intersect outside of the Young diagram $\lambda$ and there would be no such colourings which are compatible with $\lambda$. For each $i \in[l]$ in $i$-th row of $\lambda$ there are $\mathbf{q}_{i}+i-1$ boxes and $l$ of them are forbidden by non-speciality requirement; it follows that the number of choices in this third step is equal to

$$
\left(\prod_{\substack{c_{1} \in C\left(\sigma_{1}\right), c_{1} \text { is not special }}}\left[\mathbf{q}_{g_{1}\left(c_{1}\right)}+g_{1}\left(c_{1}\right)-1-l\right]\right)\left(\prod_{\substack{c_{2} \in C\left(\sigma_{2}\right), c_{2} \text { is not special }}}\left[\mathbf{q}_{g_{2}\left(c_{2}\right)}+g_{2}\left(c_{2}\right)-1-l\right]\right),
$$

where for $c_{1} \in C\left(\sigma_{1}\right), c_{2} \in C\left(\sigma_{2}\right)$ which are not special we define

$$
\begin{gathered}
g_{1}\left(c_{1}\right)=\max \left\{f_{2}\left(c_{2}\right): c_{2} \in C\left(\sigma_{2}\right), c_{2}\right. \text { is special } \\
\text { and the cycles } \left.c_{1} \text { and } c_{2} \text { intersect }\right\}, \\
g_{2}\left(c_{2}\right)=\max \left\{f_{1}\left(c_{1}\right): c_{1} \in C\left(\sigma_{1}\right), c_{1}\right. \text { is special } \\
\text { and the cycles } \left.c_{1} \text { and } c_{2} \text { intersect }\right\} .
\end{gathered}
$$


The exact form of (49) is not really important; we will use only the observation that it is a polynomial in $\mathbf{q}_{1}, \ldots, \mathbf{q}_{l}$ of degree equal to the number of non-special cycles. Since we are interested in the top-degree part of the polynomial $\Psi_{m}^{\text {spin }}\left(\mathrm{Ch}_{\pi}^{\text {spin }}\right) \in$ $\mathbb{Q}\left[\mathbf{q}_{1}, \ldots, \mathbf{q}_{l}\right]$, hence our goal is to maximize the number of such non-special cycles.

In the following it will be sometimes convenient to identify a given cycle $c \in C\left(\sigma_{1}\right) \sqcup$ $C\left(\sigma_{2}\right)$ with its support $c \subseteq[|\pi|]$ which is a non-empty subset of $[|\pi|]=\{1, \ldots,|\pi|\}$. We denote by $N \subseteq C\left(\sigma_{1}\right) \sqcup C\left(\sigma_{2}\right)$ the set of all non-special cycles. We consider an arbitrary map $s: N \rightarrow[|\pi|]$ which to any non-special cycle associates one of its elements, i.e. $f(c) \in c$ for $c \in C\left(\sigma_{1}\right) \sqcup C\left(\sigma_{2}\right)$. The map $s$ is injective; otherwise this would contradict the assumption that any two non-special cycles are disjoint. This has twofold consequences.

Firstly, the number of non-special cycles is bounded from above by $|\pi|$ so the degree of the polynomial $N_{\sigma_{1}, \sigma_{2}}(\lambda) \in \mathbb{Z}\left[\mathbf{q}_{1}, \ldots, \mathbf{q}_{l}\right]$ is bounded from above by $|\pi|$.

Secondly, if this bound on the number of non-special cycles is saturated, the map $s$ is a bijection. In this case for each $x \in[|\pi|]$ exactly one of the following two possibilities holds true: either

- $(x)$ is a non-special cycle of $\sigma_{1}$ and $x$ belongs to some special cycle of $\sigma_{2}$, or

- $(x)$ is a non-special cycle of $\sigma_{2}$ and $x$ belongs to some special cycle of $\sigma_{1}$.

The above discussion implies that the product $\sigma_{1} \sigma_{2} \in \mathfrak{S}_{|\pi|}$ can be calculated in a very simple way. Indeed, let us consider some special cycle $c$; let us say that it is a cycle of $\sigma_{1}$. Since $\sigma_{2}$ restricted to the support of $c$ is equal to the identity, the restriction of the product $\sigma_{1} \sigma_{2}$ to the support of $c$ coincides with the cycle $c$. An analogous result remains true in the case when $c$ is a special cycle of $\sigma_{2}$. In this way we proved that the product $\sigma_{1} \sigma_{2}$ coincides with the collection of the special cycles.

In this way we proved that if the number of non-special cycles achieves its maximal value $|\pi|$, each cycle of $\pi=\sigma_{1} \sigma_{2} \in \mathfrak{S}_{|\pi|}$ is either a special cycle of $\sigma_{1}$ or a special cycle of $\sigma_{2}$ and there are no other special cycles.

For example, if $\pi=\left(\pi_{1}\right) \in \mathcal{O P}$ is an odd partition which consists of only one part and

$$
\pi=\left(1,2, \ldots, \pi_{1}\right) \in \mathfrak{S}_{\pi_{1}}
$$

is the corresponding permutation, the maximal number of non-special cycles is obtained for the following two choices:

$$
\sigma_{1}=\mathrm{id}=\underbrace{(1)(2) \cdots\left(\pi_{1}\right)}_{\text {non-special cycles }}, \quad \sigma_{2}=\underbrace{\left(1,2, \ldots, \pi_{1}\right)}_{\text {special cycle }},
$$

and

$$
\sigma_{1}=\underbrace{\left(1,2, \ldots, \pi_{1}\right)}_{\text {special cycle }}, \quad \sigma_{2}=\mathrm{id}=\underbrace{(1)(2) \cdots\left(\pi_{1}\right)}_{\text {non-special cycles }} .
$$

If $j$ denotes the value of the function $f_{i}, i \in\{1,2\}$, on the unique special cycle, the homogeneous top-degree part of the corresponding summand

$$
\frac{1}{2^{\left|\sigma_{1} \vee \sigma_{2}\right|}}(-1)^{\sigma_{1}} N_{\sigma_{1}, \sigma_{2}}(\lambda)
$$

on the right-hand side of (45) is equal to

$$
\frac{1}{2} \sum_{j} \mathbf{q}_{j}^{\pi_{1}}
$$

because $\pi_{1}$ is an odd integer.

In general, for each cycle of $\pi$ there are two choices analogous to the ones above. It follows that in total there are $2^{\ell(\pi)}$ choices for $\sigma_{1}$ and $\sigma_{2}$ and which of the cycles are special. For each such a choice the total contribution of (49) is a polynomial of 
degree $|\pi|$, with the homogeneous part equal to $\frac{1}{2^{\ell(\pi)}} p_{\pi}\left(\mathbf{q}_{1}, \ldots, \mathbf{q}_{l}\right)$, a multiple of the power-sum symmetric polynomial. This concludes the proof.

\section{Proof of Theorems 1.8 And 1.9}

\section{Proof of Theorem 1.8 .}

Proof that $D^{*}(\mathbb{A}) \subseteq \Gamma$. The algebra $\mathbb{A}$ has an algebraic basis given by the functions $S_{2}, S_{3}, \ldots$ with

$$
S_{k}(\lambda)=(k-1) \iint_{(x, y) \in \lambda}(x-y)^{k-2} \mathrm{~d} x \mathrm{~d} y,
$$

see $[7]$.

The $i$-th row of a shifted Young diagram $\xi$ corresponds to two rectangles in the double $D(\xi)$. By calculating the integral over each of the rectangles it follows that

$$
\begin{aligned}
& \left(D^{*} S_{k}\right)(\xi)= \\
& \quad \frac{1}{k} \sum_{i}\left(-\xi_{i}^{k}+\left(\xi_{i}+1\right)^{k}+0^{k}-1^{k}\right)+\left(-\left(1-\xi_{i}\right)^{k}+\left(-\xi_{i}\right)^{k}+1^{k}-0^{k}\right) .
\end{aligned}
$$

It is easy to check that the polynomial in the variable $\xi_{i}$ which appears in the above formula is an odd polynomial; it follows therefore that $D^{*} S_{k} \in \mathbb{C}\left[p_{1}, p_{3}, p_{5}, \ldots\right]$ is a symmetric function which is supersymmetric. By a result of Ivanov [15, Section 6] it follows that $D^{*} S_{k} \in \Gamma$.

Proof that $D^{*}$ is surjective. We will prove a stronger result $\mathcal{G}_{k} \subseteq D^{*}\left(\mathcal{F}_{k}\right)$ below, in the proof of Theorem 1.9.

\section{Proof of Theorem 1.9.}

Proof that the family $\left(\mathcal{G}_{k}\right)$ is a filtration on the algebra $\Gamma$. Suppose that $F_{1} \in \mathcal{G}_{k_{1}}$ and $F_{2} \in \mathcal{G}_{k_{2}}$. We apply Proposition 7.4 ; for $i \in\{1,2\}$ it follows that $F_{i}(\mathbf{P} \otimes \mathbf{Q}) \in$ $\mathbb{C}\left[\mathbf{p}_{1}, \ldots, \mathbf{p}_{l}, \mathbf{q}_{1}, \ldots, \mathbf{q}_{l}\right]$ is a polynomial of degree at most $k_{i}$ hence the product $F_{1}(\mathbf{P} \otimes$ $\mathbf{Q}) F_{2}(\mathbf{P} \otimes \mathbf{Q})$ is a polynomial of degree at most $k_{1}+k_{2}$. We apply Proposition 7.4 again; it follows that $F_{1} F_{2} \in \mathcal{G}_{k_{1}+k_{2}}$. This implies that $\left(\mathcal{G}_{k}\right)$ is indeed a filtration.

Proof of the inclusion $D^{*}\left(\mathcal{F}_{k}\right) \subseteq \mathcal{G}_{k}$. Suppose $F \in \mathcal{F}_{k}$. By Proposition 7.2 and Lemma 6.1 it follows that $D^{*}(F)(\mathbf{P} \otimes \mathbf{Q})$ is a polynomial of degree at most $k$. We apply Proposition 7.4; it follows that $D^{*}(F) \in \mathcal{G}_{k}$, as required.

Proof of the inclusion $\mathcal{G}_{k} \subseteq D^{*}\left(\mathcal{F}_{k}\right)$. Let us fix an integer $k \geqslant 0$. Let $\pi \in \mathcal{O P}$ be an arbitrary odd partition such that $|\pi|+\ell(\pi) \leqslant k$. We define

$$
x_{\pi}:=-\sum_{I}\left(-\frac{1}{2}\right)^{|I|}(2|I|-3) ! ! \prod_{b \in I} \mathrm{Ch}_{\left(\pi_{i}: i \in b\right)} \in \mathcal{F}_{k},
$$

where the sum runs over all set-partitions of the set $[\ell(\pi)]$. Theorem 3.3 implies that $D^{*}\left(x_{\pi}\right)=\mathrm{Ch}_{\pi}^{\text {spin }}$; in this way we proved that $\mathrm{Ch}_{\pi}^{\text {spin }} \in D^{*}\left(\mathcal{F}_{k}\right)$. From the definition (12) of $\mathcal{G}_{k}$ it follows that $\mathcal{G}_{k} \subseteq D^{*}\left(\mathcal{F}_{k}\right)$, as required.

Acknowledgements. We thank Valentin Féray and Maciej Dołęga for several inspiring discussions.

\section{REFERENCES}

[1] Philippe Biane, Representations of symmetric groups and free probability, Adv. Math. 138 (1998), no. 1, 126-181.

[2] Characters of symmetric groups and free cumulants, in Asymptotic combinatorics with applications to mathematical physics (St. Petersburg, 2001), Lecture Notes in Math., vol. 1815, Springer, Berlin, 2003, pp. 185-200.

[3] _ On the formula of Goulden and Rattan for Kerov polynomials, Sém. Lothar. Comb. $\mathbf{5 5}(2005 / 07)$, B55d (5 pages). 
[4] Agnieszka Czyżewska-Jankowska and Piotr Śniady, Bijection between oriented maps and weighted non-oriented maps, Electron. J. Comb. 24 (2017), no. 3, 3.7 (34 pages).

[5] Dario De Stavola, Asymptotic results for representation theory, https://arxiv.org/abs/1805. 04065, 2018.

[6] Maciej Dołęga and Piotr Śniady, Gaussian fluctuations of Jack-deformed random Young diagrams, Probab. Theory Relat. Fields 174 (2019), no. 1-2, 133-176.

[7] Maciej Dołęga, Valentin Féray, and Piotr Śniady, Explicit combinatorial interpretation of Kerov character polynomials as numbers of permutation factorizations, Adv. Math. 225 (2010), no. 1, $81-120$.

[8] _ Jack polynomials and orientability generating series of maps, Sém. Lothar. Comb. 70 (2014), B70j (50 pages).

[9] Jehanne Dousse and Valentin Féray, Asymptotics for skew standard Young tableaux via bounds for characters, https://arxiv.org/abs/1710.05652, 2017.

[10] Valentin Féray, Stanley's formula for characters of the symmetric group, Ann. Comb. 13 (2010), no. 4, 453-461.

[11] Valentin Féray and Piotr Śniady, Asymptotics of characters of symmetric groups related to Stanley character formula, Ann. Math. (2) 173 (2011), no. 2, 887-906.

[12] Z Zonal polynomials via Stanley's coordinates and free cumulants, J. Algebra 334 (2011), no. $1,338-373$

[13] Ian P. Goulden and Amarpreet Rattan, An explicit form for Kerov's character polynomials, Trans. Am. Math. Soc. 359 (2007), no. 8, 3669-3685.

[14] Akihito Hora, The limit shape problem for ensembles of Young diagrams, SpringerBriefs in Mathematical Physics, vol. 17, Springer, Tokyo, 2016.

[15] Vladimir Ivanov, Gaussian limit for projective characters of large symmetric groups, J. Math. Sci., New York 121 (2004), no. 3, 2330-2344.

[16] . Plancherel measure on shifted Young diagrams, in Representation theory, dynamical systems, and asymptotic combinatorics, Amer. Math. Soc. Transl. Ser. 2, vol. 217, Amer. Math. Soc., Providence, RI, 2006, pp. 73-86.

[17] Serguei Kerov and Grigori Olshanski, Polynomial functions on the set of Young diagrams, C. R. Acad. Sci. Paris Sér. I Math. 319 (1994), no. 2, 121-126.

[18] Alexander Kleshchev, Linear and projective representations of symmetric groups, Cambridge Tracts in Mathematics, vol. 163, Cambridge University Press, Cambridge, 2005.

[19] Sergei K. Lando and Alexander K. Zvonkin, Graphs on surfaces and their applications, Encyclopaedia of Mathematical Sciences, vol. 141, Springer-Verlag, Berlin, 2004.

[20] Svante Linusson, Samu Potka, and Robin Sulzberger, On random shifted standard Young tableaux and 132-avoiding sorting networks, https://arxiv.org/abs/1804.01795, 2018.

[21] Ian Grant Macdonald, Symmetric functions and Hall polynomials, second ed., Oxford Mathematical Monographs, The Clarendon Press, Oxford University Press, New York, 1995, With contributions by A. Zelevinsky, Oxford Science Publications.

[22] Sho Matsumoto, A spin analogue of Kerov polynomials, SIGMA, Symmetry Integrability Geom. Methods Appl. 14 (2018), 053 (13 pages).

[23] Sho Matsumoto and Piotr Śniady, Stanley character formula for the spin characters of the symmetric groups, Séminaire Lotharingien de Combinatoire 82B (2019), \#1 (12 pages), Proceedings of the 31st Conference on Formal Power Series and Algebraic Combinatorics (Ljubljana).

[24] Sho Matsumoto and Piotr Śniady, Random strict partitions and random shifted tableaux, Selecta Mathematica 26 (2020), no. 1, 10, https://doi.org/10.1007/s00029-020-0535-2.

[25] Pierre-Loïc Méliot, Representation theory of symmetric groups, Discrete Mathematics and its Applications, CRC Press, Boca Raton, FL, 2017.

[26] Maxim Nazarov, Young's orthogonal form of irreducible projective representations of the symmetric group, J. Lond. Math. Soc., II. Ser. 42 (1990), no. 3, 437-451.

[27] Pasquale Petrullo and Domenico Senato, Explicit formulae for Kerov polynomials, J. Algebr. Comb. 33 (2011), no. 1, 141-151.

[28] Amarpreet Rattan and Piotr Śniady, Upper bound on the characters of the symmetric groups for balanced Young diagrams and a generalized Frobenius formula, Adv. Math. 218 (2008), no. 3, 673-695.

[29] Issai Schur, Über die Darstellung der symmetrischen und der alternierenden Gruppe durch gebrochene lineare Substitutionen, J. Reine Angew. Math. 139 (1911), 155-250.

[30] Piotr Śniady, Gaussian fluctuations of characters of symmetric groups and of Young diagrams, Probab. Theory Relat. Fields 136 (2006), no. 2, 263-297.

[31] _ Combinatorics of asymptotic representation theory, in European Congress of Mathematics, Eur. Math. Soc., Zürich, 2013, pp. 531-545. 
[32] Stanley character polynomials, in The mathematical legacy of Richard P. Stanley, Amer. Math. Soc., Providence, RI, 2016, pp. 323-334.

[33] _ Asymptotics of Jack characters, J. Comb. Theory, Ser. A 166 (2019), 91-143.

[34] Richard P. Stanley, Enumerative combinatorics. Vol. 2, Cambridge Studies in Advanced Mathematics, vol. 62, Cambridge University Press, Cambridge, 1999, With a foreword by Gian-Carlo Rota and appendix 1 by Sergey Fomin.

[35] _ Irreducible symmetric group characters of rectangular shape, Sém. Lothar. Comb. 50 (2003/04), B50d (11 pages).

[36] - A conjectured combinatorial interpretation of the normalized irreducible character values of the symmetric group, https://arxiv.org/abs/math/0606467, 2006.

[37] John R. Stembridge, Shifted tableaux and the projective representations of symmetric groups, Adv. Math. 74 (1989), no. 1, 87-134.

[38] Jinkui Wan and Weiqiang Wang, Lectures on spin representation theory of symmetric groups, Bull. Inst. Math., Acad. Sin. (N.S.) 7 (2012), no. 1, 91-164.

Sho Matsumoto, Graduate School of Science and Engineering, Kagoshima University, 1-2135, Korimoto, Kagoshima, Japan

E-mail : shom@sci.kagoshima-u.ac.jp

Piotr Śniady, Institute of Mathematics, Polish Academy of Sciences, ul. Śniadeckich 8, 00956 Warszawa, Poland

E-mail : psniady@impan.pl 